Publ. Mat. (2014), 353-394

Proceedings of New Trends in Dynamical Systems. Salou, 2012.

DOI: $10.5565 /$ PUBLMAT_Extra14_19

\title{
INVARIANT TORI IN THE LUNAR PROBLEM
}

\author{
Kenneth R. Meyer, Jesús F. Palacián, and Patricia Yanguas \\ Dedicated to Jaume Llibre on his 60th birthday
}

\begin{abstract}
Theorems on the existence of invariant KAM tori are established for perturbations of Hamiltonian systems which are circle bundle flows. By averaging the perturbation over the bundle flow one obtains a Hamiltonian system on the orbit (quotient) space by a classical theorem of Reeb. A non-degenerate critical point of the system on the orbit space gives rise to a family of periodic solutions of the perturbed system. Conditions on the critical points are given which insure KAM tori for the perturbed flow.

These general theorems are used to show that the near circular periodic solutions of the planar lunar problem are orbitally stable and are surrounded by KAM 2-tori.

For the spatial case it is shown that there are periodic solutions of two types, either near circular equatorial, that is, the infinitesimal particle moves close to the plane of the primaries following near circular trajectories and the other family where the infinitesimal particle moves along the axis perpendicular to the plane of the primaries following near rectilinear trajectories. We prove that the two solutions are elliptic and are surrounded by invariant 3-tori applying a recent theorem of $\mathrm{Han}, \mathrm{Li}$, and $\mathrm{Yi}$.

In the spatial case a second averaging is performed, and the corresponding orbit space (called the twice-reduced space) is constructed. The flow of the averaged Hamiltonian on it is given and several families of invariant 3-tori are determined using Han, Li, and Yi Theorem.
\end{abstract}

2010 Mathematics Subject Classification: 34C20, 34C25, 37J15, 37J40, 53D20, 70F10, 70K50, 70K65.

Key words: Averaging, normalization, symmetry reduction, orbit space, restricted three-body problem, planar and spatial lunar problems, invariant theory, periodic solutions, action-angle coordinates, invariant KAM tori.

\section{Introduction}

In an earlier paper [36] the authors investigated the existence, characteristic multipliers and stability of periodic solutions of a Hamiltonian vector field which is a small perturbation of a vector field tangent to the fibers of a circle bundle. Our primary examples are the planar and spatial lunar problems of celestial mechanics, i.e., the restricted three-body problem where the infinitesimal particle is close to one of the primaries. 
By averaging the perturbation over the fibers of the circle bundle one obtains a Hamiltonian system on the orbit (quotient) space of the circle bundle. We stated and proved results which have hypotheses on the reduced system and draw conclusions about the full system. Then we applied the general results to the planar and spatial lunar problems. After scaling, the lunar problem is a perturbation of the Kepler problem, which after regularization is a circle bundle flow. We found the classical near circular periodic solutions in the planar case and the near circular equatorial and certain near rectilinear periodic solutions in the spatial case. Then we computed their approximate multipliers and showed that there is a "twist". However, the twist was too degenerate to apply the classical KAM Theorem on invariant tori as stated in [2].

In this paper we prove sharper general stability theorems that show when a degenerate twist is adequate to establish invariant tori near a periodic solution. For two-degrees-of-freedom problems it is enough to appeal to the classical invariant curve theorem [28], but higher-dimensional problems require the more delicate recent result of $\mathrm{Han}, \mathrm{Li}$, and $\mathrm{Yi}[\mathbf{1 6}]$.

Then we apply these general theorems to show that the circular periodic solutions of the planar lunar problem are enclosed by invariant 2-tori, hence orbitally stable and in the spatial case that there are invariant 3-tori enclosing the periodic solutions that are near circular equatorial or near rectilinear in the vertical axis.

We also deal with the axial symmetry reduction in the spatial lunar problem. This is achieved by performing a second averaging, specifically, normalizing the argument of the node. After truncating higher-order terms the third component of the angular momentum becomes an integral of the normalized Hamiltonian and we build the corresponding orbit space called the twice-reduced space. This leads to the appearance of elliptic relative equilibria of the normalized Hamiltonian for some combinations of the parameters. This allows us to obtain invariant KAM tori related to these equilibria. Because of the degeneracy of the averaged Hamiltonian we cannot apply the usual KAM Theorems to conclude the existence of invariant tori. Again we resort to the theorem by Han, Li, and $\mathrm{Yi}[\mathbf{1 6}]$ to overcome this difficulty. Using some local action-angle coordinates that we construct specifically for the different types of elliptic relative equilibria, we establish the existence of KAM 3-tori for the Hamiltonian of the spatial restricted three-body problem in the lunar case.

The paper has five sections. In Section 2 we give the general results that will be used in subsequent sections. Some of the results of Section 2 are classic, some others are recent. Some of the results collected 
in this section appeared in [36] but Subsections 2.2, 2.3, and 2.4 are new (leaving apart Theorem 2.4). Section 3 contains the application to the planar lunar problem, leading to the existence of families of KAM 2-tori around the near circular orbits. In Section 4 we deal with the spatial case by constructing the orbit space which is a symplectic manifold of dimension four. We analyze the relative equilibria and their stability, concluding with the existence of two families of KAM 3-tori, one related to motions that are near circular equatorial and the other related with motions near rectilinear. Section 5 is devoted to a further reduction for the spatial problem. After averaging with respect to the node we truncate higher-order terms and obtain a Hamiltonian which has the third component of the angular momentum as an integral. Thus we can carry out a second reduction process, the so-called axial symmetry reduction, passing from the four-dimensional orbit space to an orbit space of dimension two, the twice-reduced space. The analysis of the flow on this space is made in detail and we can reconstruct some families of KAM 3tori using Theorem 2.4. This is achieved after constructing appropriate sets of action-angle pairs in the three types of relative equilibria that are elliptic.

The analysis performed in Sections 3 and 4 is a continuation of the work initiated in [36] but the conclusions on the existence of the invariant 2 - and 3-tori that we present in this paper are new. On the other hand part of the study in Section 5 has appeared in $[\mathbf{9}, \mathbf{2 1}, \mathbf{2 2}, \mathbf{3 2}]$ but the analysis of these papers is incomplete. A rather complete analysis was provided by Sommer [35] but, in our opinion, it is very involved so we have tried to simplify it in our presentation. Nevertheless Sommer builds a specific theorem for dealing with Hamiltonians where the perturbation appears at three different scales, making it very degenerate, and it is to her credit that she obtained a KAM-type result that applies to the spatial lunar problem which establishes the existence of new families of invariant 3-tori. Han, Li, and Yi [16] have a more general result which includes that of Sommer and it is precisely this result, Theorem 2.4, that we apply to obtain our KAM tori. Moreover we connect the results of Section 5 with those of Section 4 to clarify some points that are a bit obscure in Sommer's presentation.

\section{Perturbation theorems}

2.1. The orbit space. In this subsection we summarize some general results from our earlier paper $[\mathbf{3 6}]$. Let $(M, \Omega)$ be a symplectic manifold of dimension $2 n, \mathcal{H}_{0}: M \rightarrow \mathbb{R}$ a smooth Hamiltonian which defines a 
Hamiltonian vector field $Y_{0}=\left(d \mathcal{H}_{0}\right)^{\#}$ with symplectic flow $\phi_{0}^{t}$. Let $\mathbb{I} \subset \mathbb{R}$ be an interval such that each $h \in \mathbb{I}$ is a regular value of $\mathcal{H}_{0}$ and $\mathcal{N}_{0}(h)=\mathcal{H}_{0}^{-1}(h)$ is a compact connected circle bundle over the orbit space $B(h)$ with projection $\pi: \mathcal{N}_{0}(h) \rightarrow B(h)$. Assume that the vector field $Y_{0}$ is everywhere tangent to the fibers of $\mathcal{N}_{0}(h)$, i.e., that all the solutions of $Y_{0}$ in $\mathcal{N}_{0}(h)$ are periodic. We assume that all these periodic solutions have periods smoothly depending only on the value of the Hamiltonian, i.e., the period is a smooth function $T=T(h)$ (sometimes the dependence on $h$ will be omitted in the notation).

Now we state two of Reeb's classic Theorems [33] in more modern terminology referring to our earlier paper for proofs. Our proofs gave more insight on the Hamiltonian structure and therefore lead to further applications. The original reduction theorem is the following

Theorem 2.1. The orbit space $B$ inherits a symplectic structure $\omega$ from $(M, \Omega)$, i.e., $(B, \omega)$ is a symplectic manifold.

Now look at a perturbation of this situation. Let $\varepsilon$ be a small parameter, $\mathcal{H}_{1}: M \rightarrow \mathbb{R}$ smooth, $\mathcal{H}_{\varepsilon}=\mathcal{H}_{0}+\varepsilon \mathcal{H}_{1}, Y_{\varepsilon}=Y_{0}+\varepsilon Y_{1}=d \mathcal{H}_{\varepsilon}^{\#}$, $\mathcal{N}_{\varepsilon}(h)=\mathcal{H}_{\varepsilon}^{-1}(h)$, and $\phi_{\varepsilon}^{t}$ the flow defined by $Y_{\varepsilon}$. We shall refer to this as the full system.

Let the average of $\mathcal{H}_{1}$ be

$$
\overline{\mathcal{H}}=\frac{1}{T} \int_{0}^{T} \mathcal{H}_{1}\left(\phi_{0}^{t}\right) d t,
$$

which is a smooth function on $B(h)$, and let $\bar{\phi}^{t}$ be the flow on $B(h)$ defined by $\bar{Y}=d \overline{\mathcal{H}}^{\#}$. We refer to this as the reduced system.

A critical point of $\overline{\mathcal{H}}$ is non-degenerate if the Hessian at the critical point is non-singular.

Theorem 2.2. If $\overline{\mathcal{H}}$ has a non-degenerate critical point at $\pi(p)=\bar{p} \in B$ with $p \in \mathcal{N}_{0}$, then there are smooth functions $p(\varepsilon)$ and $T(\varepsilon)$ for $\varepsilon$ small with $p(0)=p, T(0)=T, p(\varepsilon) \in \mathcal{N}_{\varepsilon}$, and the solution of $Y_{\varepsilon}$ through $p(\varepsilon)$ is $T(\varepsilon)$-periodic.

Let the characteristic exponents of the critical point $\bar{Y}(\bar{p})$ be $\lambda_{1}, \lambda_{2}, \ldots$, $\lambda_{2 n-2}$. Then the characteristic multipliers of the periodic solution through $p(\varepsilon)$ are

$$
1,1,1+\varepsilon \lambda_{1} T+O\left(\varepsilon^{2}\right), 1+\varepsilon \lambda_{2} T+O\left(\varepsilon^{2}\right), \ldots, 1+\varepsilon \lambda_{2 n-2} T+O\left(\varepsilon^{2}\right) .
$$

That is to say, a non-degenerate critical point of the reduced system gives rise to a periodic solution of the full system. The essence of the proof of Theorem 2.2 is the existence of symplectic coordinates for a tubular neighborhood of the orbit through $p$. 
Lemma 2.1. Let $p \in \mathcal{N}_{0}(h)$, with $h \in \mathbb{I}$ fixed. Then there are symplectic coordinates $(I, \theta, y)$, valid in a tubular neighborhood of the periodic solution $\phi_{0}^{t}(p)$ of $Y_{0}(h)$, where $(I, \theta)$ are action-angle coordinates, and $y \in \mathcal{N}$, where $\mathcal{N}$ is an open neighborhood of the origin in $\mathbb{R}^{2 n-2}$. The point $p$ corresponds to $(I, \theta, y)=(0,0,0)$.

In these coordinates $\mathcal{H}_{0}$ is only a function of $I$, i.e., $\mathcal{H}_{0}=\mathcal{H}_{0}(I)$. A local cross section is $\theta=\alpha$, and a local cross section in an energy level is $\theta=\alpha, I=\beta$, where $\alpha, \beta$ are constants. In addition to that, $y \in \mathcal{N}$ are coordinates in the cross section in the energy level.

The Hamiltonian is

$$
\mathcal{H}_{\varepsilon}(I, \theta, y)=\mathcal{H}_{0}(I)+\varepsilon \mathcal{H}_{1}(I, \theta, y)=\mathcal{H}_{0}(I)+\varepsilon \overline{\mathcal{H}}(I, y)+O\left(\varepsilon^{2}\right) .
$$

2.2. Invariant 2-tori. Sometimes one can detect invariant tori using KAM Theory and at times even stability. First let us consider a simple two-degree-of-freedom system where the orbit space is two-dimensional.

Theorem 2.3. Let $n=2$ and let $p$ be as in Theorem 2.2 and Lemma 2.1. Suppose there are symplectic action-angle variables $\left(I_{1}, \theta_{1}\right)$ at $\bar{p}$ in $B$ such that

$$
\overline{\mathcal{H}}=\omega_{1} I_{1}+\varepsilon k\left(I, I_{1}\right)+O\left(\varepsilon^{2}\right),
$$

where $\omega_{1}$ is non-zero and

$$
\frac{\partial^{2} k\left(I, I_{1}\right)}{\partial I_{1}^{2}} \neq 0
$$

Then for sufficiently small $\varepsilon>0$ encircling the periodic solutions given in Theorem 2.2 there are invariant KAM tori of dimension 2. In particular the periodic solutions are orbitally stable.

Proof: Take the cross section at $p$ given by Lemma 2.1 with $\theta=0$ and $I=0$. The first return time is $T_{\varepsilon}=T+O\left(\varepsilon^{2}\right)$.

On $B$ the equations are

$$
\dot{I}_{1}=O\left(\varepsilon^{2}\right), \quad \dot{\theta}_{1}=-\omega_{1}-\varepsilon \frac{\partial k}{\partial I_{1}}\left(0, I_{1}\right)+O\left(\varepsilon^{2}\right) .
$$

Integrate these equations by a time $T_{\varepsilon}$ to find the period map $P:\left(I_{1}, \theta_{1}\right) \rightarrow$ $\left(I_{1}^{\prime}, \theta_{1}^{\prime}\right)$ where

$$
I_{1}^{\prime}=I_{1}+O\left(\varepsilon^{2}\right), \quad \theta_{1}^{\prime}=\theta_{1}-\omega_{1} T-\varepsilon T \frac{\partial k}{\partial I_{1}}\left(0, I_{1}\right)+O\left(\varepsilon^{2}\right) .
$$

By (3) the twist assumption of Moser's invariant curve Theorem [28] holds which implies the existence of arbitrarily small invariant curves encircling $p$ in the cross section. These invariant curves produce invariant 
tori in the phase space of the full system defined by (1) and so the periodic solutions are orbitally stable.

2.3. Higher-order tori. First we state the result of Han, Li, and $\mathrm{Yi}[\mathbf{1 6}]$, because it gives invariant tori in systems with a degenerate twist of the type we encounter in the lunar problem and other similar problems [26]. Refer to those papers for comments about other results that yield KAM tori in degenerate situations.

Starting with a Hamiltonian system of the form

(4) $\mathcal{H}(I, \varphi, \varepsilon)=h_{0}\left(I^{n_{0}}\right)+\varepsilon^{m_{1}} h_{1}\left(I^{n_{1}}\right)+\cdots+\varepsilon^{m_{a}} h_{a}\left(I^{n_{a}}\right)+\varepsilon^{m_{a}+1} p(I, \varphi, \varepsilon)$,

where $(I, \varphi) \in \mathbb{R}^{n} \times \mathbb{T}^{n}$ are action-angle variables with the standard symplectic structure $d I \wedge d \varphi$, and $\varepsilon>0$ is a sufficiently small parameter. The Hamiltonian $\mathcal{H}$ is real analytic in $(I, \phi, \varepsilon)$ and in particular $p$ is smooth in $\varepsilon$. The parameters $a, m, n_{i}(i=0,1, \ldots, a)$ and $m_{j}(j=$ $1,2, \ldots, a)$, are positive integers satisfying $n_{0} \leq n_{1} \leq \cdots \leq n_{a}=n$, $m_{1} \leq m_{2} \leq \cdots \leq m_{a}=m, I^{n_{i}}=\left(I_{1}, \ldots, I_{n_{i}}\right)$, for $i=1,2, \ldots, a$.

The Hamiltonian $\mathcal{H}(I, \varphi, \varepsilon)$ is considered in a bounded closed region $Z \times \mathbb{T}^{n} \times\left[0, \varepsilon^{*}\right] \subset \mathbb{R}^{n} \times \mathbb{T}^{n} \times\left[0, \varepsilon^{*}\right]$ for some fixed $\varepsilon^{*}$ with $0<\varepsilon^{*}<1$. For each $\varepsilon$ the integrable part of $\mathcal{H}$,

$$
X_{\varepsilon}(I)=h_{0}\left(I^{n_{0}}\right)+\varepsilon^{m_{1}} h_{1}\left(I^{n_{1}}\right)+\cdots+\varepsilon^{m_{a}} h_{a}\left(I^{n_{a}}\right),
$$

admits a family of invariant $n$-tori $T_{\zeta}^{\varepsilon}=\{\zeta\} \times \mathbb{T}^{n}$ with linear flows $\left\{x_{0}+\omega^{\varepsilon}(\zeta) t\right\}$, where, for each $\zeta \in Z, \omega^{\varepsilon}(\zeta)=\nabla X_{\varepsilon}(\zeta)$ is the frequency vector of the $n$-torus $T_{\zeta}^{\varepsilon}$ and $\nabla$ is the gradient operator. When $\omega^{\varepsilon}(\zeta)$ is non-resonant, the flow on the $n$-torus $T_{\zeta}^{\varepsilon}$ becomes quasi-periodic with slow and fast frequencies of different scales. We refer the integrable part $X_{\varepsilon}$ and its associated tori $\left\{T_{\zeta}^{\varepsilon}\right\}$ as the intermediate Hamiltonian and tori, respectively.

Let $\bar{I}^{n_{i}}=\left(I_{n_{i-1}+1}, \ldots, I_{n_{i}}\right), i=0,1, \ldots, a$ (where $n_{-1}=0$, hence $\left.\bar{I}^{n_{0}}=I^{n_{0}}\right)$, and define

$$
\Omega=\left(\nabla_{\bar{I}^{n_{0}}} h_{0}\left(I^{n_{0}}\right), \ldots, \nabla_{\bar{I}^{n_{a}}} h_{n_{a}}\left(I^{n_{a}}\right)\right)
$$

such that for each $i=0,1, \ldots, a, \nabla_{\bar{I}^{n_{i}}}$ denotes the gradient with respect to $\bar{I}^{n_{i}}$.

The following theorem gives the right setting in which one can ensure the persistence of KAM tori for Hamiltonians like (4). 
Theorem 2.4 (Han, Li, and Yi [16]). Let $\delta$ be given with $0<\delta<1 / 5$. Assume there is a positive integer $s$ such that

$$
\operatorname{Rank}\left\{\partial_{I}^{\alpha} \Omega(I)|0 \leq| \alpha \mid \leq s\right\}=n, \quad \forall I \in Z .
$$

Then there exists an $\varepsilon_{0}>0$ and a family of Cantor sets $Z_{\varepsilon} \subset Z, 0<\varepsilon<$ $\varepsilon_{0}$, such that each $\zeta \in Z_{\varepsilon}$ corresponds to a real analytic, invariant, quasiperiodic $n$-torus $\bar{T}_{\zeta}^{\varepsilon}$ of the Hamiltonian (4) which is slightly deformed from the intermediate $n$-torus $T_{\zeta}^{\varepsilon}$. The measure of $Z \backslash Z_{\varepsilon}$ is $O\left(\varepsilon^{\delta / s}\right)$ and the family $\left\{\bar{T}_{\zeta}^{\varepsilon}: \zeta \in Z_{\varepsilon}, 0<\varepsilon<\varepsilon_{0}\right\}$ varies Whitney smoothly.

The beauty of this level of generalization is that if the conditions of Theorem 2.4 apply to the Hamiltonian on the orbit space then conditions of Theorem 2.4 apply to the full system. This follows by using Lemma 2.1 in conjunction with Han, Li, and Yi's Theorem.

However for one of our applications we require something less, namely

Theorem 2.5. Let $p$ be as in Theorem 2.2 and suppose there are symplectic action-angle variables $\left(I_{1}, \ldots, I_{n-1}, \theta_{1}, \ldots, \theta_{n-1}\right)$ at $\bar{p}$ in $B$ such that

$$
\overline{\mathcal{H}}=\sum_{k=1}^{n-1} \omega_{k} I_{k}+\frac{\varepsilon^{j}}{2} \sum_{k=1}^{n-1} \sum_{l=1}^{n-1} C_{k l} I_{k} I_{l}+O\left(\varepsilon^{j+1}\right),
$$

where $j \geq 0$, the $\omega_{k}$ 's are non-zero, the coefficients $C_{k l}$ 's are independent of $\varepsilon$ and satisfy $C_{k l}=C_{l k}$, and all the cubic and higher-order terms in $I_{1}, \ldots, I_{n-1}$ are included in $O\left(\varepsilon^{j+1}\right)$.

Assume that $\operatorname{det} C_{k l} \neq 0$. That is, assume the system has been put into Birkhoff normal form and a "twist" condition is satisfied. Furthermore, assume $d T / d h \neq 0$, i.e., assume the period varies with $\mathcal{H}_{0}$ in a nontrivial way.

Then for sufficiently small $\varepsilon>0$ near the periodic solution given in Theorem 2.2 there are invariant KAM tori of dimension $n$.

Proof: The numbering system of our notation and that of $\mathrm{Han}, \mathrm{Li}$, and $\mathrm{Yi}$ as stated in Theorem 2.4 are slightly different. Let the $I_{j}$ 's be $I, I_{1}, \ldots, I_{n-1}$ where $I$ is as in Lemma 2.1 and $I_{1}, \ldots, I_{n-1}$ are as above. If you think of $I$ as $I_{0}$ then we have shifted the indexes down by 1.

Combining (1) with (2) gives

(7) $\mathcal{H}_{\varepsilon}(I, \theta, y)=\mathcal{H}_{0}(I)+\varepsilon \sum_{k=1}^{n-1} \omega_{k} I_{k}+\frac{\varepsilon^{j+1}}{2} \sum_{k=1}^{n-1} \sum_{l=1}^{n-1} C_{k l} I_{k} I_{l}+O\left(\varepsilon^{j+2}\right)$. 
The assumption that $d T / d h \neq 0$ is equivalent to $\partial^{2} \mathcal{H}_{0} / \partial I^{2} \neq 0$. Now condition (5) is satisfied since it is clear that the rank of

$$
\partial_{I}^{\alpha} \Omega(I)=\left[\begin{array}{cc}
\partial^{2} \mathcal{H}_{0} / \partial I^{2} & 0 \\
0 & C
\end{array}\right]
$$

is $n$ where $C$ is the matrix $\left[C_{k l}\right]$ and $s=1$.

2.4. Regularization. In our subsequent applications of Theorems 2.4 and 2.5, the term $\mathcal{H}_{0}$ will be the Hamiltonian of the Kepler problem. For the consideration of circular solutions (in the planar and the spatial cases) or equatorial solutions (in the spatial case) of the restricted threebody problem, classical Poincaré-like coordinates assure that $d T / d h \neq 0$, but for the rectilinear ones which pass close to collisions we will need to use regularized coordinates. The Kepler problem has a removable singularity due to collisions of the infinitesimal with the primary. Removing the singularity is called regularization. It is important in our study of collision and near-collision periodic solutions and their related invariant tori.

Moser [29] shows that the $n$-dimensional Kepler problem can be regularized in the sense that there is a symplectomorphism that takes the Kepler flow for a fixed negative energy level to the geodesic flow onto the unit tangent bundle of the punctured $n$-sphere, $\hat{S}^{n}$, punctured at the north pole. The geodesic flow of the unit sphere over the north pole corresponds to the collision orbits and by adding it back in effect adds the collisions in a regular flow.

Let $\mathcal{E}$ be the whole negative energy region of the Kepler problem, the elliptic region, and let $T^{+} \hat{S}^{n}$ be the tangent bundle of the punctured sphere minus the zero section. Ligon and Schaaf [24] show how to transform canonically the whole elliptic region $\mathcal{E}$ to the bundle $T^{+} \hat{S}^{n}$. This transformation takes the usual Hamiltonian of the Kepler problem to a Hamiltonian $D$ on $T^{+} \hat{S}^{n}$. Hamiltonian $D$ extends naturally to $T^{+} S^{n}$ thus making effective the regularization of the Kepler problem for all negative energies. Also see $[\mathbf{1 0}]$ and the simpler approach given by Heckman and de Laat in [18].

For the Kepler problem or the flow defined by $D$ the period is constant on energy levels and $d T / d h \neq 0$ even at the regularized collision orbits. This is precisely what we need to assure the existence of families of invariant 2-tori in Section 3 and invariant 3-tori in Sections 3 and 4, even around the near rectilinear periodic solutions in Subsection 4.3. 


\section{The planar lunar problem}

3.1. The averaged Hamiltonian for the planar problem. Here we briefly summarize the normalization and reduction as given in [36]. For us the lunar problem is the restricted three-body problem where the infinitesimal is close to one of the primaries. We start with the Hamiltonian of the planar restricted three-body problem given by:

$$
\begin{aligned}
\mathcal{H}=\frac{1}{2}\left(y_{1}^{2}+y_{2}^{2}\right)- & \left(x_{1} y_{2}-x_{2} y_{1}\right) \\
& -\frac{\mu}{\sqrt{\left(x_{1}-1+\mu\right)^{2}+x_{2}^{2}}}-\frac{1-\mu}{\sqrt{\left(x_{1}+\mu\right)^{2}+x_{2}^{2}}} .
\end{aligned}
$$

First we perform the linear change from $y_{2}$ and $x_{1}$ to $y_{2}-\mu$ and $x_{1}-\mu$ respectively to bring one primary to the origin. Then, we introduce a small parameter $\varepsilon$ by replacing $y=\left(y_{1}, y_{2}\right)$ by $\varepsilon^{-1}(1-\mu)^{1 / 3} y$ and $x=\left(x_{1}, x_{2}\right)$ by $\varepsilon^{2}(1-\mu)^{1 / 3} x$. By doing so we restrict $\mathcal{H}$ to the case where the infinitesimal is moving around one of the primaries. This change is symplectic with multiplier $\varepsilon^{-1}(1-\mu)^{-2 / 3}$, thus $\mathcal{H}$ must be replaced by $\varepsilon^{-1}(1-\mu)^{-2 / 3} \mathcal{H}$.

Next we scale time by dividing $t$ by $\varepsilon^{3}$ and multiplying $\mathcal{H}$ by $\varepsilon^{3}$. Then we expand the resulting Hamiltonian in powers of $\varepsilon$ to get

$$
\begin{aligned}
\mathcal{H}_{\varepsilon}=\frac{1}{2}\left(y_{1}^{2}+y_{2}^{2}\right) & -\frac{1}{\sqrt{x_{1}^{2}+x_{2}^{2}}} \\
& -\varepsilon^{3}\left(x_{1} y_{2}-x_{2} y_{1}\right)+\frac{1}{2} \varepsilon^{6} \mu\left(-2 x_{1}^{2}+x_{2}^{2}\right)+O\left(\varepsilon^{8}\right) .
\end{aligned}
$$

The zeroth-order term is the Hamiltonian of the Kepler problem and the $O\left(\varepsilon^{3}\right)$ term is due to the rotating coordinates. It is not until $O\left(\varepsilon^{6}\right)$ that the other primary influences the motion. This applies for the whole treatment of the lunar planar and spatial restricted three-body problems in this section and in Sections 4 and 5.

To construct this flow on the orbit space of (9) we used in [36] a blend of polar and Delaunay coordinates so that the Hamiltonian is ready for the elimination of the mean anomaly $\ell$ to high order by means of the normalization of Delaunay $[\mathbf{1 2}, \mathbf{1 3}]$. This normalization is effectively the average of the perturbation over the periodic motions of the Kepler problem.

The orbit space for the regularized planar Kepler problem is a 2sphere $S^{2}$ [29]. A coordinate system for the reduced space is $\mathbf{a}=\mathbf{G}+$ $L A$, where $\mathbf{G}$ is the angular momentum vector, $L$ is the square root 
of the semimajor axis, and $A$ is the perigee vector ${ }^{1}$. One has $A=$ $e(\cos g, \sin g, 0)$ and then $a_{1}=e \cos g, a_{2}=e \sin g$ and $a_{3}=G$, with $G$ the third component of $\mathbf{G}$, and $e=\sqrt{1-G^{2} / L^{2}}$ the eccentricity. One can check that $|\mathbf{a}|=L$ and the vector a uniquely determines an orbit of the Kepler problem on the energy level $h=-1 /\left(2 L^{2}\right)$. Each point on the sphere $a_{1}^{2}+a_{2}^{2}+a_{3}^{2}=L^{2}$ corresponds to a bounded orbit of the Kepler problem. The points $(0,0, \pm L)$ correspond to circular motions, the circle $a_{3}=0$ corresponds to collision motions, and the other points on the sphere correspond to elliptic motions. The complement of $(0,0, \pm L) \cup$ $\left\{a_{3}=0\right\}$ is the orbit space of the elliptic domain $\mathcal{E}$.

We now have

$$
\overline{\mathcal{H}}=-a_{3}-\frac{3}{4} \varepsilon^{3} \mu L^{2}\left(3 a_{1}^{2}-2 a_{2}^{2}\right)+O\left(\varepsilon^{5}\right) .
$$

We note that this Hamiltonian is well defined and smooth on the exceptional set $(0,0, \pm L) \cup\left\{a_{3}=0\right\}$ so (10) is the Hamiltonian on the full orbit space $S^{2}$.

The Hamiltonian (10) has two non-degenerate critical points, a maximum at $\mathbf{a}=(0,0,-L)$ and a minimum at $\mathbf{a}=(0,0, L)$, which correspond to near circular periodic solutions of the planar restricted three-body problem of period $T(\varepsilon)=T+O\left(\varepsilon^{3}\right)$. These are the classical Hill's orbits of the restricted problem, which are the continuation of the circular solutions of the Kepler problem (see [7, 25] and the references therein). The maximum gives the prograde orbit and the minimum the retrograde one.

Since $\overline{\mathcal{H}}=-a_{3}+\cdots$ the linearized equations about $(0,0, \pm L)$ are $\dot{a}_{1}=a_{2}, \dot{a}_{2}=-a_{1}$, and so the characteristic exponents at these critical points are $\pm i$.

Thus, these near circular periodic solutions are elliptic with characteristic multipliers $1,1,1+\varepsilon^{3} T i+O\left(\varepsilon^{6}\right)$ and $1-\varepsilon^{3} T i+O\left(\varepsilon^{6}\right)$.

To see if Theorem 2.3 applies at $(0,0, \pm L)$ we need several changes of variables. We start by moving the equilibria $(0,0, \pm L)$ to the origin of a coordinate system. Therefore, we define

$$
\bar{a}_{1}=a_{1}, \quad \bar{a}_{2}=a_{2}, \quad \bar{a}_{3}=a_{3} \mp L,
$$

\footnotetext{
${ }^{1}$ The vector $A$ points to the perigee which is often attributed to Laplace, Runge, Lenz and others. But since Newton and Kepler knew that perigee was fixed we choose this neutral name.
} 
and then we introduce (local) symplectic coordinates $Q$ and $P$ as:

$$
\begin{aligned}
& Q=\sqrt{2} \frac{L \bar{a}_{1}}{\sqrt{2 L \pm \bar{a}_{3}}}=\sqrt{2(L \mp G)} \cos g \\
& P= \pm \sqrt{2} \frac{L \bar{a}_{2}}{\sqrt{2 L \pm \bar{a}_{3}}}= \pm \sqrt{2(L \mp G)} \sin g .
\end{aligned}
$$

By recalling that $(\ell, g, L, G)$ are symplectic variables, it is straightforward to check that $(Q, P)$ are symplectic. These coordinates are valid in the hemispheres $\pm a_{3}>0$ (i.e., $\pm G<L$ ).

Now, to write $\overline{\mathcal{H}}$ in these coordinates first note that

$$
\frac{1}{2}\left(Q^{2}+P^{2}\right)=L \mp G=L \mp a_{3},
$$

and also

$$
a_{1}^{2}=\frac{Q^{2}}{2 L^{2}}\left(L \pm a_{3}\right), \quad a_{2}^{2}=\frac{P^{2}}{2 L^{2}}\left(L \pm a_{3}\right) .
$$

Making this change of variables and omitting additive constants gives

$$
\overline{\mathcal{H}}= \pm \frac{1}{2}\left(Q^{2}+P^{2}\right)-\frac{3}{16} \varepsilon^{3} \mu\left(2 P^{2}-3 Q^{2}\right)\left(Q^{2}+P^{2}-4 L\right)+O\left(\varepsilon^{5}\right) .
$$

Change to action-angle variables by

$$
Q=\sqrt{2 I_{1}} \cos \theta_{1}, \quad P=\sqrt{2 I_{1}} \sin \theta_{1}
$$

to get

$$
\overline{\mathcal{H}}= \pm I_{1}-\frac{3}{4} \varepsilon^{3} \mu I_{1}\left(2 L-I_{1}\right)\left(-2+5 \cos ^{2} \theta_{1}\right)+O\left(\varepsilon^{5}\right),
$$

then average over $\theta_{1}$ to get

$$
\overline{\mathcal{H}}= \pm I_{1}-\frac{3}{8} \varepsilon^{3} \mu I_{1}\left(2 L-I_{1}\right)+O\left(\varepsilon^{5}\right) .
$$

To apply Theorem 2.3 make the identification $k=-\frac{3}{8} \mu I_{1}\left(2 L-I_{1}\right)$. Now

$$
\frac{\partial^{2} k}{\partial I_{1}^{2}}=\frac{3}{4} \mu,
$$

and it does not vanish. So the hypotheses of Theorem 2.3 hold (after replacing $\varepsilon^{3}$ by $\delta$ and considering $\delta$ the small parameter of Section 2). Moreover, the measure of the frequencies of the invariant curves appearing in the proof of Theorem 2.3 is positive. Also, we can be assured that there are invariant 2-tori surrounding the periodic solutions arbitrarily close to it. Thus we have proved the following 
Proposition 3.1. The near circular periodic solutions of the planar lunar problem are orbitally stable and enclosed by invariant KAM 2-tori for small enough $\varepsilon$.

\section{The spatial lunar problem}

4.1. The averaged Hamiltonian for the spatial problem. Again we summarize results from [36]. As in the planar case we start with the Hamiltonian of the spatial problem given in the rotating frame by

$$
\begin{aligned}
\mathcal{H}=\frac{1}{2}\left(y_{1}^{2}+y_{2}^{2}+y_{3}^{2}\right)-\left(x_{1} y_{2}-x_{2} y_{1}\right) & \\
& -\frac{\mu}{\sqrt{\left(x_{1}-1+\mu\right)^{2}+x_{2}^{2}+x_{3}^{2}}}-\frac{1-\mu}{\sqrt{\left(x_{1}+\mu\right)^{2}+x_{2}^{2}+x_{3}^{2}}} .
\end{aligned}
$$

Then we move one primary to the origin, introduce a small parameter $\varepsilon$ that ensures that the infinitesimal is close to a primary, scale the Hamiltonian as in the planar case, and expand the Hamiltonian in powers of $\varepsilon$. Then, we obtain:

$$
\begin{aligned}
\mathcal{H}_{\varepsilon}=\frac{1}{2}( & \left.y_{1}^{2}+y_{2}^{2}+y_{3}^{2}\right)-\frac{1}{\sqrt{x_{1}^{2}+x_{2}^{2}+x_{3}^{2}}} \\
& \quad-\varepsilon^{3}\left(x_{1} y_{2}-x_{2} y_{1}\right)+\frac{1}{2} \varepsilon^{6} \mu\left(-2 x_{1}^{2}+x_{2}^{2}+x_{3}^{2}\right)+O\left(\varepsilon^{8}\right) .
\end{aligned}
$$

Next we express the Hamiltonian in mixed polar-nodal and Delaunay coordinates and eliminate the mean anomaly to a fixed order using Lie transformations $[\mathbf{1 1}, \mathbf{2 5}]$. The normalized Hamiltonian reads

$$
\begin{aligned}
\mathcal{H}_{\varepsilon}=-\frac{1}{2 L^{2}}-\varepsilon^{3} N & \\
+\frac{1}{16} \varepsilon^{6} \mu L^{4}( & \left(2+3 e^{2}\right)\left(1-3 c^{2}-3\left(1-c^{2}\right) \cos (2 \nu)\right) \\
& +30 c e^{2} \sin (2 g) \sin (2 \nu) \\
& \left.-15 e^{2} \cos (2 g)\left(1-c^{2}+\left(1+c^{2}\right) \cos (2 \nu)\right)\right)+O\left(\varepsilon^{8}\right),
\end{aligned}
$$

where $G=|\mathbf{G}|, N$ is the projection of $\mathbf{G}$ onto the axis $x_{3}$ (note that $N$ coincides with $G$ in the planar version of the Delaunay coordinates) and $\nu$ is the argument of the node, e.g. the angular coordinate conjugate to $N$; finally $c=N / G$ represents the cosine of the inclination angle, $I$, of the orbital plane with respect to the equatorial plane and it is enough to consider $I \in[0, \pi]$. The transformed Hamiltonian, after truncating higher-order terms, depends on the two angles $g$ and $\nu$ and their associated momenta $G$ and $N$ respectively, whereas $L$ is an integral of motion. 
Applying reduction theory, once higher-order terms have been dropped, the Hamiltonian $\overline{\mathcal{H}}$ is defined on the orbit space which is the four-dimensional manifold $S^{2} \times S^{2}$ [29]. A similar argument to that given for the planar Kepler problem in Subsection 3.1 shows that $S^{2} \times S^{2}$ is indeed the orbit space of the regularized spatial Kepler problem since collision orbits can be studied in this space as we show below.

We can use the set of variables given by $\mathbf{a}=\left(a_{1}, a_{2}, a_{3}\right)$ and $\mathbf{b}=$ $\left(b_{1}, b_{2}, b_{3}\right)$ with the constraints $a_{1}^{2}+a_{2}^{2}+a_{3}^{2}=b_{1}^{2}+b_{2}^{2}+b_{3}^{2}=L^{2}$ to parameterize $S^{2} \times S^{2}$, where $\mathbf{a}=\mathbf{G}+L A$ and $\mathbf{b}=\mathbf{G}-L A$. The vector $A$ represents the three-dimensional perigee vector. Note that the expressions of the components of $A$ and $\mathbf{a}$ in terms of the Delaunay coordinates are not the same as in the planar case, see $[\mathbf{8 , 3 1}$. A representation of the space $S^{2} \times S^{2}$ is given in Figure 1: on the left, the red point represents the vector a on the sphere $|\mathbf{a}|=L$ whereas on the right one, the green point accounts for the vector $\mathbf{b}$ on the sphere $|\mathbf{b}|=L$.
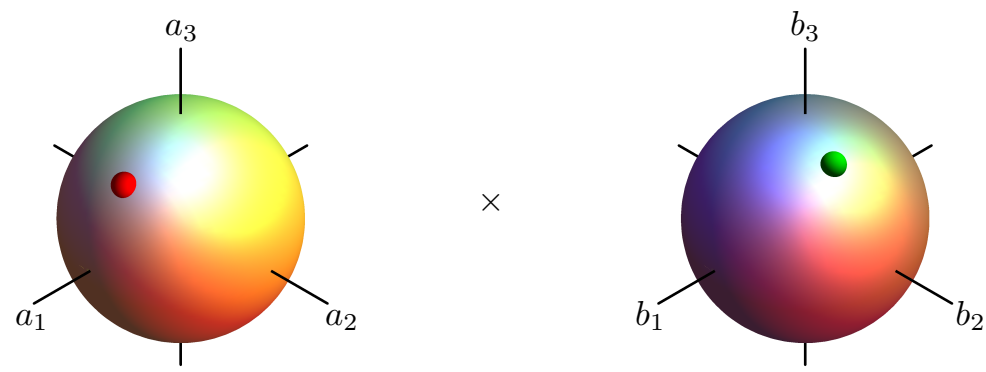

FiguRE 1. A single motion in the orbit space $S^{2} \times S^{2}$ is given through the two points on their corresponding spheres.

As $2 G=\left(\left(a_{1}+b_{1}\right)^{2}+\left(a_{2}+b_{2}\right)^{2}+\left(a_{3}+b_{3}\right)^{2}\right)^{1 / 2}$, then $G=0$ if and only if $a_{1}+b_{1}=a_{2}+b_{2}=a_{3}+b_{3}=0, a_{1}^{2}+a_{2}^{2}+a_{3}^{2}=L^{2}$ and $b_{1}^{2}+b_{2}^{2}+b_{3}^{2}=L^{2}$. So the subset of $S^{2} \times S^{2}$ defined by $\mathcal{R}=\left\{(\mathbf{a},-\mathbf{a}) \in \mathbb{R}^{6} \mid a_{1}^{2}+a_{2}^{2}+a_{3}^{2}=L^{2}\right\}$ is a two-dimensional set consisting of collision trajectories. As in Delaunay variables the circular orbits satisfy $G=L$ this implies that $a_{1}=b_{1}$, $a_{2}=b_{2}$ and $a_{3}=b_{3}$. Thus the circular orbits define the two-dimensional set given by $\mathcal{C}=\left\{(\mathbf{a}, \mathbf{a}) \in \mathbb{R}^{6} \mid a_{1}^{2}+a_{2}^{2}+a_{3}^{2}=L^{2}\right\}$. On the other hand equatorial motions satisfy $G=|N|$ and are given by the twodimensional set $\mathcal{E} q=\left\{(\mathbf{a}, \mathbf{b}) \in \mathbb{R}^{6} \mid a_{1}^{2}+a_{2}^{2}+a_{3}^{2}=L^{2}, b_{1}=-a_{1}, b_{2}=\right.$ $\left.-a_{2}, b_{3}=a_{3}\right\}$. The use of the variables $\mathbf{a}$ and $\mathbf{b}$ extends the use of 
the Delaunay variables, as we can include equatorial, circular and linear trajectories $[\mathbf{3 1}, \mathbf{3 6}]$. Finally, the other points on $S^{2} \times S^{2}$ correspond to elliptic motions of the spatial Kepler problem.

After several simplifications and manipulations over $\mathcal{H}_{\varepsilon}$, the normal form Hamiltonian expressed in the invariants $\mathbf{a}$ and $\mathbf{b}$ is

$$
\begin{aligned}
\overline{\mathcal{H}}= & -\frac{1}{2}\left(a_{3}+b_{3}\right) \\
& -\frac{3}{8} \varepsilon^{3} \mu L^{2}\left(a_{1}^{2}-a_{2}^{2}-a_{3}^{2}+b_{1}^{2}-b_{2}^{2}-b_{3}^{2}-4 a_{1} b_{1}\right. \\
& \left.+2 a_{2} b_{2}+2 a_{3} b_{3}\right)+O\left(\varepsilon^{5}\right) .
\end{aligned}
$$

Now, as the Hamiltonian starts as $\overline{\mathcal{H}}=-\frac{1}{2}\left(a_{3}+b_{3}\right)+\cdots$ it has a non-degenerate maximum at $(\mathbf{a}, \mathbf{b})=(0,0,-L, 0,0,-L)$ and a nondegenerate minimum at $(\mathbf{a}, \mathbf{b})=(0,0, L, 0,0, L)$, which by Reeb's Theorem 2.2 correspond to elliptic periodic solutions of the spatial restricted three-body problem (12) of period $T(\varepsilon)=T+O\left(\varepsilon^{3}\right)$. These are the near circular equatorial solutions (also called near circular coplanar solutions as the small particle moves on the plane determined by the motion of the two primaries) already encountered in the planar case. The Hamiltonian $\overline{\mathcal{H}}$ also has two non-degenerate critical points of index 2 at $(\mathbf{a}, \mathbf{b})=(0,0, \pm L, 0,0, \mp L)$ which correspond to the near rectilinear motions whose projection in the coordinate space leads to periodic orbits in the vertical axis $x_{3}$. They are indeed the near rectilinear trajectories found by Belbruno $[\mathbf{3}]$ for small $\mu$.

One sees that the characteristic exponents of all critical points of $\bar{Y}$ are $\pm i$ (double).

Thus, the characteristic multipliers of the four families of periodic solutions of the system defined by the Hamiltonian (12) are 1, 1, 1+ $\varepsilon^{3} T i, 1+\varepsilon^{3} T i, 1-\varepsilon^{3} T i, 1-\varepsilon^{3} T i$ plus terms of order $O\left(\varepsilon^{6}\right)$.

4.2. The circular equatorial points $(0,0, \pm \boldsymbol{L}, \mathbf{0}, \mathbf{0}, \pm \boldsymbol{L})$. We first move the Hamiltonian to the origin by

$$
a_{1}=\bar{a}_{1}, \quad a_{2}=\bar{a}_{2}, \quad a_{3}=\bar{a}_{3} \pm L, \quad b_{1}=\bar{b}_{1}, \quad b_{2}=\bar{b}_{2}, \quad b_{3}=\bar{b}_{3} \pm L,
$$

then we change variables by

$$
\begin{array}{ll}
Q_{1}=\frac{\bar{a}_{2}}{\sqrt{2 L \pm \bar{a}_{3}}}, & Q_{2}=\frac{\bar{b}_{2}}{\sqrt{2 L \pm \bar{b}_{3}}}, \\
P_{1}=\mp \frac{\bar{a}_{1}}{\sqrt{2 L \pm \bar{a}_{3}}}, & P_{2}=\mp \frac{\bar{b}_{1}}{\sqrt{2 L \pm \bar{b}_{3}}},
\end{array}
$$


with inverse

$$
\begin{array}{lll}
\bar{a}_{1}=\mp P_{1} \sqrt{2 L-P_{1}^{2}-Q_{1}^{2}}, & \bar{a}_{2}=Q_{1} \sqrt{2 L-P_{1}^{2}-Q_{1}^{2}}, \quad \bar{a}_{3}=\mp\left(P_{1}^{2}+Q_{1}^{2}\right), \\
\bar{b}_{1}=\mp P_{2} \sqrt{2 L-P_{2}^{2}-Q_{2}^{2}}, & \bar{b}_{2}=Q_{2} \sqrt{2 L-P_{2}^{2}-Q_{2}^{2}}, \quad \bar{b}_{3}=\mp\left(P_{2}^{2}+Q_{2}^{2}\right) .
\end{array}
$$

The change is canonical, with $Q_{1}$ and $Q_{2}$ as coordinates and $P_{1}$ and $P_{2}$ as their associated momenta.

The resulting Hamiltonian is obtained after writing $\overline{\mathcal{H}}$ in terms of the $Q_{i}$ 's and $P_{i}$ 's and dropping constant terms, so

$$
\begin{aligned}
\overline{\mathcal{H}}= \pm & \frac{1}{2}\left(P_{1}^{2}+Q_{1}^{2}\right) \pm \frac{1}{2}\left(P_{2}^{2}+Q_{2}^{2}\right) \\
-\frac{3}{4} \varepsilon^{3} \mu L^{2}( & L\left(P_{1}^{2}+P_{2}^{2}\right)-L\left(Q_{1}^{2}+Q_{2}^{2}\right) \\
& -\left(2 P_{1} P_{2}-Q_{1} Q_{2}\right) \sqrt{2 L-P_{1}^{2}-Q_{1}^{2}} \sqrt{2 L-P_{2}^{2}-Q_{2}^{2}} \\
& \left.-\left(P_{2}^{2}-Q_{1}^{2}\right)\left(P_{2}^{2}+Q_{2}^{2}\right)-P_{1}^{2}\left(P_{1}^{2}-P_{2}^{2}+Q_{1}^{2}-Q_{2}^{2}\right)\right)+O\left(\varepsilon^{5}\right) .
\end{aligned}
$$

The Hamiltonian $\overline{\mathcal{H}}$ is valid in a neighborhood of $(0,0, \pm L, 0,0, \pm L)$.

Now we scale by $\bar{Q}_{j}=\varepsilon^{-3 / 2} Q_{j}$ and $\bar{P}_{j}=\varepsilon^{-3 / 2} P_{j}$ for $j \in\{1,2\}$. The canonical structure is preserved by dividing $\overline{\mathcal{H}}$ by $\varepsilon^{3}$. After expansion of this Hamiltonian in powers of $\varepsilon$ we obtain

$$
\begin{aligned}
\overline{\mathcal{H}}= & \pm \frac{1}{2}\left(\bar{P}_{1}^{2}+\bar{Q}_{1}^{2}\right) \pm \frac{1}{2}\left(\bar{P}_{2}^{2}+\bar{Q}_{2}^{2}\right) \\
& -\frac{3}{4} \varepsilon^{3} \mu L^{3}\left(\bar{P}_{1}^{2}+\bar{P}_{2}^{2}-4 \bar{P}_{1} \bar{P}_{2}-\bar{Q}_{1}^{2}-\bar{Q}_{2}^{2}+2 \bar{Q}_{1} \bar{Q}_{2}\right) \\
& +\frac{3}{8} \varepsilon^{6} \mu L^{2}\left(2\left(\bar{P}_{1}^{4}-\bar{P}_{1}^{3} \bar{P}_{2}-\bar{P}_{1}^{2} \bar{P}_{2}^{2}-\bar{P}_{1} \bar{P}_{2}^{3}+\bar{P}_{2}^{4}\right)+\left(\bar{P}_{1}^{2}+\bar{P}_{2}^{2}\right) \bar{Q}_{1} \bar{Q}_{2}\right. \\
& \left.+2\left(\bar{P}_{1}^{2}-\bar{P}_{1} \bar{P}_{2}+\bar{P}_{2}^{2}\right)\left(\bar{Q}_{1}^{2}-\bar{Q}_{2}^{2}\right)+\bar{Q}_{1} \bar{Q}_{2}\left(\bar{Q}_{1}-\bar{Q}_{2}\right)^{2}\right)+O\left(\varepsilon^{8}\right) .
\end{aligned}
$$

For $(0,0, L, 0,0, L)$ the eigenvalues associated with the linear differential equation given through the quadratic part of $\overline{\mathcal{H}}$ are

$$
\pm \sqrt{1+2 \bar{\varepsilon}} i= \pm \omega_{1} i, \quad \pm \sqrt{1-2 \bar{\varepsilon}-24 \bar{\varepsilon}^{2}} i= \pm \omega_{2} i
$$


with $\bar{\varepsilon}=\frac{3}{4} \varepsilon^{3} \mu L^{3}$ and $\omega_{1}>1>\omega_{2}>0$. For the point $(0,0,-L, 0,0,-L)$ the eigenvalues are

$$
\pm \sqrt{1-2 \bar{\varepsilon}} i= \pm \omega_{1} i, \quad \pm \sqrt{1+2 \bar{\varepsilon}-24 \bar{\varepsilon}^{2}} i= \pm \omega_{2} i
$$

In this case $\omega_{2}>1>\omega_{1}>0$. We remark that if $\varepsilon=0$, then $\omega_{1}=\omega_{2}=1$, thus the quadratic part of $\overline{\mathcal{H}}$ is in $1:-1$ resonance. So we keep $\varepsilon$ small but positive so that we can apply KAM Theory. As a consequence $\omega_{1}$ and $\omega_{2}$ are close to 1 but different from it.

The eigenvectors related to $\omega_{1}$ and $\omega_{2}$ form a basis of $\mathbb{R}^{4}$, thus the quadratic part of $\overline{\mathcal{H}}$ is brought into normal form through a canonical change of variables. This linear change must be applied to $\overline{\mathcal{H}}$. The columns of the matrix are the eigenvectors scaled so that the matrix is symplectic. After defining the new variables by $\left(q_{1}, q_{2}, p_{1}, p_{2}\right)$ the quadratic part of $\overline{\mathcal{H}}$ becomes

$$
\pm \omega_{1} i q_{1} p_{1} \pm \omega_{2} i q_{2} p_{2} .
$$

The values of the frequencies $\omega_{1}$ and $\omega_{2}$ are given in (16) if the quadratic part is $\omega_{1} i q_{1} p_{1}+\omega_{2} i q_{2} p_{2}$, whereas if the quadratic part is $-\omega_{1} i q_{1} p_{1}-$ $\omega_{2} i q_{2} p_{2}$ we take the frequencies from (17). From now on when referring to $(0,0, L, 0,0, L)$ we assume that $\omega_{1}$ and $\omega_{2}$ are as in $(16)$, and when we study the point $(0,0,-L, 0,0,-L)$ we take the frequencies from (17).

We introduce

$$
\begin{array}{ll}
q_{1}=\sqrt{I_{1}}\left(\cos \varphi_{1}-i \sin \varphi_{1}\right), & q_{2}=\sqrt{I_{2}}\left(\cos \varphi_{2}-i \sin \varphi_{2}\right), \\
p_{1}=\sqrt{I_{1}}\left(\sin \varphi_{1}-i \cos \varphi_{1}\right), & p_{2}=\sqrt{I_{2}}\left(\sin \varphi_{2}-i \cos \varphi_{2}\right),
\end{array}
$$

and the change satisfies $d q_{1} \wedge d p_{1}+d q_{2} \wedge d p_{2}=d I_{1} \wedge d \varphi_{1}+d I_{2} \wedge d \varphi_{2}$. This transforms the quadratic terms of $\overline{\mathcal{H}}$ into $\pm \omega_{1} I_{1} \pm \omega_{2} I_{2}$, while the quartic terms are converted into a finite Fourier series in $\varphi_{1}$ and $\varphi_{2}$ whose coefficients are homogeneous quadratic polynomials in $I_{1}$ and $I_{2}$.

Now we average $\overline{\mathcal{H}}$ over $\varphi_{1}$ and $\varphi_{2}$, obtaining

$$
\begin{aligned}
\overline{\mathcal{H}}= & \pm \omega_{1} I_{1} \pm \omega_{2} I_{2}-\frac{\left(\omega_{1}^{2}-1\right)^{2}\left(\omega_{1}^{2}+3\right)}{24 \mu L^{4} \omega_{1}^{2}} I_{1}^{2} \\
& +\frac{\left(\omega_{1}^{2}-1\right)^{2}\left(21 \omega_{1}^{2}-13\right)}{6 \mu L^{4} \omega_{1} \omega_{2}} I_{1} I_{2} \\
& -\frac{\left(\omega_{1}^{2}-1\right)^{2}\left(24 \omega_{1}^{4}-119 \omega_{1}^{2}+91\right)}{24 \mu L^{4} \omega_{2}^{2}} I_{2}^{2}+\cdots .
\end{aligned}
$$

The coefficients of $I_{1}, I_{2}, I_{1}^{2}, I_{2}^{2}$, and $I_{1} I_{2}$ may be expressed in terms of $\bar{\varepsilon}$, and expanding them in powers of $\bar{\varepsilon}$ around 0 yields expressions such that the leading terms of the coefficients of $I_{1}$ and $I_{2}$ are independent 
of $\bar{\varepsilon}$ while the coefficients of $I_{1}^{2}, I_{2}^{2}$, and $I_{1} I_{2}$ are factorized by $\bar{\varepsilon}^{2}$. The generating functions computed in the averaging process in the two cases are enormous, but they are finite Fourier series in the angles $\varphi_{1}$ and $\varphi_{2}$.

At this point we can compute the determinant of the Hessian associated with $\overline{\mathcal{H}}$. First we calculate the constraint relating $\omega_{1}$ with $\omega_{2}$ through $\bar{\varepsilon}$ using (16) or (17), obtaining

$$
\omega_{2}=\sqrt{\left(2 \omega_{1}^{2}-1\right)\left(4-3 \omega_{1}^{2}\right)} .
$$

We end up with the same expression for the points $(0,0, L, 0,0, L)$ and $(0,0,-L, 0,0,-L)$, which is

$\operatorname{det}\left[\begin{array}{cc}\frac{\partial^{2} \overline{\mathcal{H}}}{\partial I_{1}^{2}} & \frac{\partial^{2} \overline{\mathcal{H}}}{\partial I_{1} \partial I_{2}} \\ \frac{\partial^{2} \overline{\mathcal{H}}}{\partial I_{2} \partial I_{1}} & \frac{\partial^{2} \overline{\mathcal{H}}}{\partial I_{2}^{2}}\end{array}\right]=\frac{\left(\omega_{1}^{2}-1\right)^{4}\left(24 \omega_{1}^{6}-1811 \omega_{1}^{4}+1918 \omega_{1}^{2}-403\right)}{144 \mu^{2} L^{8} \omega_{1}^{2} \omega_{2}^{2}}+\cdots$.

The determinant vanishes when $\omega_{1} \in\{0.53692 \ldots, 0.88488 \ldots, 1,8.62479$

$\ldots\}$, however $\omega_{1}$ is near 1 (either above or below, but it never reaches this value as $\varepsilon$ cannot be zero). Next we express the coefficients of $\overline{\mathcal{H}}$ in terms of $\varepsilon$, divide the result by $\varepsilon^{6}$ as the quadratic terms of $\overline{\mathcal{H}}$ start at that order, compute the Hessian, and we obtain:

$$
\operatorname{det}\left[\begin{array}{cc}
\frac{\partial^{2} \overline{\mathcal{H}}}{\partial I_{1}^{2}} & \frac{\partial^{2} \overline{\mathcal{H}}}{\partial I_{1} \partial I_{2}} \\
\frac{\partial^{2} \overline{\mathcal{H}}}{\partial I_{2} \partial I_{1}} & \frac{\partial^{2} \overline{\mathcal{H}}}{\partial I_{2}^{2}}
\end{array}\right]=-\frac{153}{16} \mu^{2} L^{4}+O\left(\varepsilon^{3}\right)
$$

Thus Theorem 2.5 applies after setting $\delta=\varepsilon^{6}$, using $\delta$ as the small parameter of (6), then $j=1$.

According to $[\mathbf{1 6}]$ one has that the measure of the set omitted from the established invariant tori related to Theorem 2.4 is of order $O\left(\varepsilon^{b}\right)$ with $b=\sum_{i=1}^{a} m_{i}\left(n_{i}-n_{i-1}\right)$. In this case, we need to write down the Hamiltonian normal form $\mathcal{H}_{\varepsilon}$ arranged in powers of $\varepsilon$. This is achieved after putting the frequencies $\omega_{i}$ 's in terms of $\varepsilon$, undoing the scalings (excepting the stretching of coordinates passing from $Q_{j}$ 's and $P_{j}$ 's to $\bar{Q}_{j}$ 's and $\bar{P}_{j}$ 's as we want that the normal form will be valid in a neighborhood of the points $(0,0, \pm L, 0,0, \pm L))$, expanding the resulting expression in 
powers of $\varepsilon$ and incorporating the dropped terms to get $\overline{\mathcal{H}}$. We get

$$
\begin{aligned}
\mathcal{H}_{\varepsilon}= & -\frac{1}{2 L^{2}} \mp \varepsilon^{3} L \pm \varepsilon^{6}\left(I_{1}+I_{2}\right)+\frac{3}{4} \varepsilon^{9} \mu L^{3}\left(I_{1}-I_{2}\right) \\
& -\frac{3}{32} \varepsilon^{12} \mu L^{2}\left(4\left(I_{1}^{2}-8 I_{1} I_{2}-I_{2}^{2}\right) \pm 3 \mu L^{4}\left(I_{1}+25 I_{2}\right)\right)+O\left(\varepsilon^{15}\right) .
\end{aligned}
$$

From (18) we infer that $a=4, m_{1}=3, m_{2}=6, m_{3}=9, m_{4}=12$, $n_{0}=n_{1}=1, n_{2}=n_{3}=n_{4}=3$, therefore $b=12$, besides $s=1$. Thus, we conclude

Proposition 4.1. There are families of invariant KAM 3-tori around the near circular coplanar periodic solutions of the full system introduced by the Hamiltonian (12). These invariant tori form a majority in the sense that the measure of the complement of their union is of order $O\left(\varepsilon^{12}\right)$.

Although we do not know about the non-linear stability of the families of periodic solutions, we can say something more about the stability of the equilibria $(0,0, \pm L, 0,0, \pm L)$ of the reduced system on the base space. Since the Hamiltonian $\overline{\mathcal{H}}$ is positive or negative definite at these points, the classical theorem already known to Dirichlet $[\mathbf{1 4}, \mathbf{2 5}]$ applies.

Proposition 4.2. The relative equilibrium points $(0,0, \pm L, 0,0, \pm L)$ are stable on the reduced space $S^{2} \times S^{2}$.

4.3. The rectilinear points $(0,0, \pm L, 0,0, \mp L)$. After moving the origin to the point of interest through

$$
a_{1}=\bar{a}_{1}, \quad a_{2}=\bar{a}_{2}, \quad a_{3}=\bar{a}_{3} \pm L, \quad b_{1}=\bar{b}_{1}, \quad b_{2}=\bar{b}_{2}, \quad b_{3}=\bar{b}_{3} \mp L,
$$

we introduce the local symplectic coordinates

$$
\begin{array}{ll}
Q_{1}=\frac{\bar{a}_{2}}{\sqrt{2 L \pm \bar{a}_{3}}}, \quad Q_{2}=\frac{\bar{b}_{2}}{\sqrt{2 L \mp \bar{b}_{3}}}, \\
P_{1}=\mp \frac{\bar{a}_{1}}{\sqrt{2 L \pm \bar{a}_{3}}}, & P_{2}= \pm \frac{\bar{b}_{1}}{\sqrt{2 L \mp \bar{b}_{3}}},
\end{array}
$$

with inverse

$$
\begin{array}{lll}
\bar{a}_{1}=\mp P_{1} \sqrt{2 L-P_{1}^{2}-Q_{1}^{2}}, & \bar{a}_{2}=Q_{1} \sqrt{2 L-P_{1}^{2}-Q_{1}^{2}}, & \bar{a}_{3}=\mp\left(P_{1}^{2}+Q_{1}^{2}\right), \\
\bar{b}_{1}= \pm P_{2} \sqrt{2 L-P_{2}^{2}-Q_{2}^{2}}, & \bar{b}_{2}=Q_{2} \sqrt{2 L-P_{2}^{2}-Q_{2}^{2}}, \quad \bar{b}_{3}= \pm\left(P_{2}^{2}+Q_{2}^{2}\right) .
\end{array}
$$


The resulting Hamiltonian is obtained after putting $\overline{\mathcal{H}}$ in terms of the $Q_{i}$ 's and $P_{i}$ 's and omitting constant terms. We get

$$
\begin{aligned}
\overline{\mathcal{H}}= \pm \frac{1}{2}\left(P_{1}^{2}+Q_{1}^{2}\right) \mp \frac{1}{2}\left(P_{2}^{2}+Q_{2}^{2}\right) \\
-\frac{3}{4} \varepsilon^{3} \mu L^{2}\left(3 L\left(P_{1}^{2}+P_{2}^{2}\right)+L\left(Q_{1}^{2}+Q_{2}^{2}\right)\right. \\
+\left(2 P_{1} P_{2}+Q_{1} Q_{2}\right) \sqrt{2 L-P_{1}^{2}-Q_{1}^{2}} \sqrt{2 L-P_{2}^{2}-Q_{2}^{2}} \\
\left.-\left(P_{2}^{2}+Q_{1}^{2}\right)\left(P_{2}^{2}+Q_{2}^{2}\right)-P_{1}^{2}\left(P_{1}^{2}+P_{2}^{2}+Q_{1}^{2}+Q_{2}^{2}\right)\right)+O\left(\varepsilon^{5}\right) .
\end{aligned}
$$

The Hamiltonian $\overline{\mathcal{H}}$ is valid in a neighborhood of the points $(0,0, \pm L$, $0,0, \mp L)$.

Next we scale variables through the change $\bar{Q}_{j}=\varepsilon^{-3 / 2} Q_{j}$ and $\bar{P}_{j}=$ $\varepsilon^{-3 / 2} P_{j}$ for $j \in\{1,2\}$. To make the change canonical we must divide $\overline{\mathcal{H}}$ by $\varepsilon^{3}$. Expanding this Hamiltonian in powers of $\varepsilon$ (and keeping the same name for it) we obtain the Hamiltonian:

$$
\begin{aligned}
\overline{\mathcal{H}}= & \pm \frac{1}{2}\left(\bar{P}_{1}^{2}+\bar{Q}_{1}^{2}\right) \mp \frac{1}{2}\left(\bar{P}_{2}^{2}+\bar{Q}_{2}^{2}\right) \\
- & \frac{3}{4} \varepsilon^{3} \mu L^{3}\left(3\left(\bar{P}_{1}^{2}+\bar{P}_{2}^{2}\right)+4 \bar{P}_{1} \bar{P}_{2}+\bar{Q}_{1}^{2}+\bar{Q}_{2}^{2}+2 \bar{Q}_{1} \bar{Q}_{2}\right) \\
+ & \frac{3}{8} \varepsilon^{6} \mu L^{2}\left(2\left(\bar{P}_{1}^{4}+\bar{P}_{1}^{3} \bar{P}_{2}+\bar{P}_{1}^{2} \bar{P}_{2}^{2}+\bar{P}_{1} \bar{P}_{2}^{3}+\bar{P}_{2}^{4}\right)\right. \\
& +2 \bar{P}_{2}\left(\bar{P}_{1}+\bar{P}_{2}\right) \bar{Q}_{1}^{2}+\left(\bar{P}_{1}^{2}+\bar{P}_{2}^{2}\right) \bar{Q}_{1} \bar{Q}_{2} \\
& \left.+2\left(\bar{P}_{1}^{2}+\bar{P}_{1} \bar{P}_{2}+\bar{P}_{2}^{2}\right) \bar{Q}_{2}^{2}+\bar{Q}_{1} \bar{Q}_{2}\left(\bar{Q}_{1}+\bar{Q}_{2}\right)^{2}\right)+O\left(\varepsilon^{8}\right) .
\end{aligned}
$$

The eigenvalues associated with the linear vector field given through the quadratic part of $\overline{\mathcal{H}}$ are the expressions

$$
\begin{aligned}
& \pm \sqrt{1+20 \bar{\varepsilon}^{2}+2 \sqrt{5} \bar{\varepsilon} \sqrt{3+20 \bar{\varepsilon}^{2}}} i= \pm \omega_{1} i, \\
& \pm \sqrt{1+20 \bar{\varepsilon}^{2}-2 \sqrt{5} \bar{\varepsilon} \sqrt{3+20 \bar{\varepsilon}^{2}}} i= \pm \omega_{2} i
\end{aligned}
$$

where $\bar{\varepsilon}$ stands for $\frac{3}{4} \varepsilon^{3} \mu L^{3}$ and $\omega_{1}>1>\omega_{2}>0$. Note that $\omega_{1}=\omega_{2}=1$ when $\varepsilon=0$, and the quadratic part of $\overline{\mathcal{H}}$ is in $1:-1$ resonance. However when $\varepsilon \neq 0$ the eigenvalues are distinct. 
Thus, the relative equilibria of the reduced system are parametrically stable and therefore the corresponding periodic solutions of the full system (12) dealing with the near rectilinear motions are elliptic.

We keep $\varepsilon$ small but positive so that we may perform further normalization. By doing so, both $\omega_{1}$ and $\omega_{2}$ remain close to 1 but different from it. As the corresponding set of eigenvectors forms a basis of $\mathbb{R}^{4}$, the quadratic part of $\overline{\mathcal{H}}$ may be brought into normal form through a canonical change of variables. This linear change has to be applied to $\overline{\mathcal{H}}$. The columns of the transformation matrix are the eigenvectors related to $\pm \omega_{1} i$ and $\pm \omega_{2} i$ multiplied by scale constants chosen to make the change symplectic. We do not give the explicit expression for this change because it is lengthy and the procedure is standard; see for instance $[\mathbf{5}, \mathbf{2 3}]$. Denoting the new variables by $\left(q_{1}, q_{2}, p_{1}, p_{2}\right)$ and using the same name for the Hamiltonian, its quadratic part becomes in both cases

$$
-\omega_{1} i q_{1} p_{1}+\omega_{2} i q_{2} p_{2} \text {. }
$$

Next we introduce action-angle variables $\left(I_{1}, I_{2}, \varphi_{1}, \varphi_{2}\right)$ by means of

$$
\begin{array}{ll}
q_{1}=\sqrt{I_{1}}\left(\cos \varphi_{1}-i \sin \varphi_{1}\right), & q_{2}=\sqrt{I_{2}}\left(\cos \varphi_{2}-i \sin \varphi_{2}\right), \\
p_{1}=\sqrt{I_{1}}\left(\sin \varphi_{1}-i \cos \varphi_{1}\right), & p_{2}=\sqrt{I_{2}}\left(\sin \varphi_{2}-i \cos \varphi_{2}\right) .
\end{array}
$$

It is easy to check that $d q_{1} \wedge d p_{1}+d q_{2} \wedge d p_{2}=d I_{1} \wedge d \varphi_{1}+d I_{2} \wedge d \varphi_{2}$. This transformation brings the quadratic terms of $\overline{\mathcal{H}}$ to $-\omega_{1} I_{1}+\omega_{2} I_{2}$, while its quartic terms are converted into a finite Fourier series in $\varphi_{1}$ and $\varphi_{2}$ whose coefficients are homogeneous quadratic polynomials in $I_{1}$ and $I_{2}$. We do not give the Hamiltonian because it is enormous.

Now we average $\overline{\mathcal{H}}$ over $\varphi_{1}$ and $\varphi_{2}$, and we obtain:

$$
\begin{aligned}
\overline{\mathcal{H}}= & -\omega_{1} I_{1}+\omega_{2} I_{2}+\frac{\left(7 \omega_{1}^{6}+13 \omega_{1}^{4}+13 \omega_{1}^{2}+3\right)\left(\omega_{1}^{2}-1\right)^{2}}{30 \mu L^{4} \omega_{1}^{2}\left(\omega_{1}^{2}+2\right)^{2}\left(2 \omega_{1}^{2}+1\right)} I_{1}^{2} \\
& -\frac{2\left(\omega_{1}^{2}-1\right)^{2}\left(\omega_{1}^{4}-14 \omega_{1}^{2}-5\right)\left(2 \omega_{2}^{2}+1\right)}{135 \mu L^{4} \omega_{1}\left(\omega_{1}^{2}+2\right)^{2} \omega_{2}} I_{1} I_{2} \\
& +\frac{\left(7 \omega_{2}^{6}+13 \omega_{2}^{4}+13 \omega_{2}^{2}+3\right)\left(\omega_{2}^{2}-1\right)^{2}}{30 \mu L^{4} \omega_{2}^{2}\left(\omega_{2}^{2}+2\right)^{2}\left(2 \omega_{2}^{2}+1\right)} I_{2}^{2}+\cdots .
\end{aligned}
$$

The coefficients of $I_{1}^{2}, I_{2}^{2}$, and $I_{1} I_{2}$ may be expressed in terms of $\bar{\varepsilon}$, and after expanding them in powers of $\bar{\varepsilon}$, one obtains a formula starting in $\bar{\varepsilon}^{2}$, while the leading terms of the coefficients of $I_{1}$ and $I_{2}$ do not depend on $\bar{\varepsilon}$. The generating function responsible for this averaging step 
is too big to be reproduced here, but it is a finite Fourier series in the angles $\varphi_{1}$ and $\varphi_{2}$.

Now we can compute the determinant of the Hessian associated with $\overline{\mathcal{H}}$. Using the constraint which relates $\omega_{1}$ and $\omega_{2}$ through (19) given by

$$
\omega_{2}=\sqrt{\frac{4-\omega_{1}^{2}}{2 \omega_{1}^{2}+1}},
$$

we get

$\operatorname{det}\left[\begin{array}{cc}\frac{\partial^{2} \overline{\mathcal{H}}}{\partial I_{1}^{2}} & \overline{\partial^{2} \overline{\mathcal{H}}} \\ \frac{\partial^{2} \overline{\mathcal{H}}}{\partial I_{2} \partial I_{2}} & \frac{\partial^{2} \overline{\mathcal{H}}}{\partial I_{2}^{2}}\end{array}\right]=\frac{\left(\omega_{1}^{2}-1\right)^{6}\left(7 \omega_{1}^{8}-28 \omega_{1}^{6}-534 \omega_{1}^{4}-604 \omega_{1}^{2}-137\right)}{225 \mu^{2} L^{8} \omega_{1}^{2}\left(\omega_{1}^{2}-4\right)\left(\omega_{1}^{2}+2\right)^{4}\left(2 \omega_{1}^{2}+1\right)^{2}}+\cdots$

which does not vanish since the (positive) real roots of the determinant occur for $\omega_{1}=1$ or $\omega_{1}=3.37369 \ldots$, but as $\varepsilon$ does not vanish $\omega_{1}$ remains greater than 1 .

Now we need to express the coefficients of $\overline{\mathcal{H}}$ in terms of $\varepsilon$. First of all we use (19) to put $\omega_{1}$ and $\omega_{2}$ in terms of $\varepsilon$ and divide the result by $\varepsilon^{6}$ as the quadratic terms of $\overline{\mathcal{H}}$ start at that order. Then we compute the Hessian, getting

$$
\operatorname{det}\left[\begin{array}{cc}
\frac{\partial^{2} \overline{\mathcal{H}}}{\partial I_{1}^{2}} & \frac{\partial^{2} \overline{\mathcal{H}}}{\partial I_{1} \partial I_{2}} \\
\frac{\partial^{2} \overline{\mathcal{H}}}{\partial I_{2} \partial I_{1}} & \frac{\partial^{2} \overline{\mathcal{H}}}{\partial I_{2}^{2}}
\end{array}\right]=\frac{405}{4} \varepsilon^{6} \mu^{4} L^{10}+O\left(\varepsilon^{12}\right) .
$$

Thus Theorem 2.5 cannot be applied directly as the determinant above is factorized by $\varepsilon^{6}$. Nevertheless we can still apply Theorem 2.4. Undoing the scalings introduced to obtain $\overline{\mathcal{H}}$ as function of the actions $I_{1}$ and $I_{2}$ (excepting the stretching of variables that define $\bar{Q}_{i}$ 's and $\bar{P}_{i}$ 's in terms of $Q_{i}$ 's and $P_{i}$ 's), incorporating the terms dropped in the process, expressing the $\omega_{i}$ 's in terms of $\varepsilon$, expanding the whole Hamiltonian in terms of $\varepsilon$ we end up with

$$
\begin{aligned}
\mathcal{H}_{\varepsilon}= & -\frac{1}{2 L^{2}}+\varepsilon^{6}\left(-I_{1}+I_{2}\right)-\frac{3 \sqrt{15}}{4} \varepsilon^{9} \mu L^{3}\left(I_{1}+I_{2}\right) \\
& +\frac{3}{32} \varepsilon^{12} \mu L^{2}\left(16\left(I_{1}+I_{2}\right)^{2}+15 \mu L^{4}\left(-I_{1}+I_{2}\right)\right)+O\left(\varepsilon^{15}\right) .
\end{aligned}
$$


The Hamiltonian (20) is valid near the points $(0,0, \pm L, 0,0, \mp L)$. Then Theorem 2.4 can be applied taking $a=3, m_{1}=6, m_{2}=9, m_{3}=$ $12, n_{0}=1, n_{1}=n_{2}=n_{3}=3, I^{n_{0}}=(L), I^{n_{1}}=I^{n_{2}}=I^{n_{3}}=$ $\left(L, I_{1}, I_{2}\right), \bar{I}^{n_{0}}=(L), \bar{I}^{n_{1}}=\bar{I}^{n_{2}}=\bar{I}^{n_{3}}=\left(I_{1}, I_{2}\right)$. If $h_{0}$ is the Keplerian Hamiltonian in Delaunay variables and $h_{1}, h_{2}$, and $h_{3}$ denote respectively the terms of (20) factorized by $\varepsilon^{6}, \varepsilon^{9}$, and $\varepsilon^{12}$, the vector of frequencies has dimension six and is given explicitly by

$$
\begin{aligned}
\Omega(I)= & \left(\frac{\partial h_{0}}{\partial L}, \frac{\partial h_{1}}{\partial I_{1}}, \frac{\partial h_{1}}{\partial I_{2}}, \frac{\partial h_{2}}{\partial I_{1}}, \frac{\partial h_{2}}{\partial I_{2}}, \frac{\partial h_{3}}{\partial I_{1}}, \frac{\partial h_{3}}{\partial I_{2}}\right) \\
= & \left(\frac{1}{L^{3}},-1,1,-\frac{3 \sqrt{15}}{4} \mu L^{3},-\frac{3 \sqrt{15}}{4} \mu L^{3},\right. \\
& \left.3\left(I_{1}+I_{2}\right) \mu L^{2}-\frac{45}{32} \mu^{2} L^{6}, 3\left(I_{1}+I_{2}\right) \mu L^{2}+\frac{45}{32} \mu^{2} L^{6}\right)
\end{aligned}
$$

and the $4 \times 6$-matrix with rows $\Omega(I), \partial \Omega(I) / \partial L, \partial \Omega(I) / \partial I_{1}$ and $\partial \Omega(I) / \partial I_{2}$ has rank three, leading to the existence of the invariant tori of dimension three.

We apply Remark 2 of $[\mathbf{1 6}$, p. 1422] to estimate the measure of the surviving invariant tori (in the sense of the persistence of the KAM tori that remain when higher-order terms are added). According to [16] one has that the measure of the set omitted from the established invariant tori is of order $O\left(\varepsilon^{b}\right)$ with $b=\sum_{i=1}^{a} m_{i}\left(n_{i}-n_{i-1}\right)=12$. As we only needed the first-order partial derivatives in (5) we have $s=1$. Thus, we conclude

Proposition 4.3. There are families of invariant KAM 3-tori around the near rectilinear periodic solutions of the full system defined through the Hamiltonian (12). These invariant tori form a majority in the sense that the measure of the complement of their union is of order $O\left(\varepsilon^{12}\right)$.

These invariant tori are the generalization of the punctured 2-tori in the lunar case of the planar restricted three-body problem studied by Chenciner and Llibre [4].

Contrarily to the planar case we do not know the non-linear stability of the periodic solutions. However, we can say something about the stability of the equilibria $(0,0, \pm L, 0,0, \mp L)$ of the reduced system on the base space.

For the analysis of the stability of these equilibria we use Arnold's Theorem $[\mathbf{2 7}, \mathbf{3 4}]$. We fix $\varepsilon$ small and positive. We need to find $\overline{\mathcal{H}}_{4}$, i.e., the quartic terms of $\overline{\mathcal{H}}$ as functions of the $q_{i}$ 's, $p_{i}$ 's, in other words, the 
quadratic terms of $\overline{\mathcal{H}}$ as functions of $I_{1}$ and $I_{2}$, and then compute

$$
\begin{aligned}
\overline{\mathcal{H}}_{4}\left(\omega_{2}, \omega_{1}\right) & =\frac{\left(\omega_{1}^{2}-1\right)^{2}\left(\omega_{1}^{12}-16 \omega_{1}^{10}+66 \omega_{1}^{8}-268 \omega_{1}^{6}-275 \omega_{1}^{4}-132 \omega_{1}^{2}-24\right)}{15 \mu L^{4} \omega_{1}^{2}\left(\omega_{1}^{2}-4\right)\left(2 \omega_{1}^{4}+5 \omega_{1}^{2}+2\right)^{2}} \\
& =6 \varepsilon^{6} \mu L^{2}+O\left(\varepsilon^{9}\right) .
\end{aligned}
$$

Since this term does not vanish for $\omega_{1}$ close to (but larger than) 1, Arnold's Theorem applies, and thus

Proposition 4.4. The linear equilibria points $(0,0, \pm L, 0,0, \mp L)$ are stable on the orbit space $S^{2} \times S^{2}$.

Even when $\overline{\mathcal{H}}_{4}\left(\omega_{2}, \omega_{1}\right)$ has $\varepsilon^{6}$ as a factor, Arnold's Theorem in the form given in $[\mathbf{3 0}]$ applies (see also $[\mathbf{2 5}, \mathbf{2 7}]$ ). Indeed in theorems presented in $[\mathbf{2 5}, \mathbf{2 7}, \mathbf{3 0}]$, although $\varepsilon$ does not appear in the statements, it is introduced in the proofs by scaling. The proofs rely on the final theorem in Moser's classic paper [28] where the invariant curve theorem allows small twists, i.e., terms multiplied by $\varepsilon^{a}$ with $a>0$. This idea was already used in the proof of Theorem 2.3.

In Subsection 4.2 we could have used the symplectic coordinates introduced in $[\mathbf{2 0}]$, which are defined to deal with near circular equatorial motions in $S^{2} \times S^{2}$. The computations carried out with this set of variables are a bit shorter than the ones we have applied in Subsection 4.2 but we have wanted to maintain a similar set to the one used for the linear motions here.

\section{The second reduction in the spatial lunar problem}

5.1. Elimination of the node and the twice-reduced space. Next we eliminate the argument of the node by averaging the Hamiltonian (14) with respect to the angle $\nu$. Our aim is to get more insight in the dynamics of the spatial lunar restricted three-body problem, obtaining new invariant 3-tori of the Hamiltonian (12). The normalized Hamiltonian has been studied by several authors, see the references $[\mathbf{9}, \mathbf{3 2}, \mathbf{3 5}]$, and we summarize the main results here.

After applying the Lie transformation method $[\mathbf{1 1}, \mathbf{2 5}]$ with the aim of averaging the angle $\nu$, we end up with the following Hamiltonian

$$
\begin{aligned}
\mathcal{H}_{\varepsilon}= & -\frac{1}{2 L^{2}}-\varepsilon^{3} N \\
& +\frac{1}{16} \varepsilon^{6} \mu L^{4} \\
& \left(1-3 c^{2}\right)\left(5-3 \eta^{2}\right) \\
& \left.\quad-15\left(1-c^{2}\right)\left(1-\eta^{2}\right) \cos (2 g)\right)+O\left(\varepsilon^{8}\right),
\end{aligned}
$$

where $\eta=\sqrt{1-e^{2}}=G / L$. 
After truncating higher-order terms (21) defines a one-degree-of-freedom system which is invariant with respect to the Keplerian symmetry and the axial symmetry, or equivalently, the actions $L$ and $N$ become approximate integrals of motion. Thus we can reduce the Hamiltonian $\mathcal{H}_{\varepsilon}$ after constructing the corresponding orbit space.

The algebra of polynomials on $S^{2} \times S^{2}$ invariant under the action associated with this reduction is generated by the $\pi_{i}$ 's where

$$
\begin{array}{lll}
\pi_{1}=a_{1}^{2}+a_{2}^{2}, & \pi_{2}=a_{1} b_{2}-a_{2} b_{1}, & \pi_{3}=a_{3}, \\
\pi_{4}=b_{1}^{2}+b_{2}^{2}, & \pi_{5}=a_{1} b_{1}+a_{2} b_{2}, & \pi_{6}=b_{3},
\end{array}
$$

together with the constraints

$$
\pi_{1}+\pi_{3}^{2}=L^{2}, \quad \pi_{4}+\pi_{6}^{2}=L^{2}, \quad \pi_{2}^{2}+\pi_{5}^{2}=\pi_{1} \pi_{4} .
$$

Taking the map

$$
\pi_{N}: S^{2} \times S^{2} \longrightarrow\{N\} \times \mathbb{R}^{3}:(\mathbf{a}, \mathbf{b}) \mapsto\left(N, \tau_{1}, \tau_{2}, \tau_{3}\right) \equiv(N, \tau),
$$

where

$$
\tau_{1}=\frac{1}{2}\left(\pi_{3}-\pi_{6}\right), \quad \tau_{2}=\pi_{2}, \quad \tau_{3}=\pi_{5}
$$

we define the invariants $\tau_{1}, \tau_{2}$, and $\tau_{3}$ in terms of $\mathbf{a}$ and $\mathbf{b}$ as

$$
\tau_{1}=\frac{1}{2}\left(a_{3}-b_{3}\right), \quad \tau_{2}=a_{1} b_{2}-a_{2} b_{1}, \quad \tau_{3}=a_{1} b_{1}+a_{2} b_{2} .
$$

The constraints (23) are used to define the corresponding orbit space. The space $\mathcal{T}_{L, N}$, called twice-reduced space, is defined as the image of $S^{2} \times S^{2}$ by $\pi_{N}$, that is,

$$
\begin{aligned}
\mathcal{T}_{L, N} & =\pi_{N}\left(S^{2} \times S^{2}\right) \\
& =\left\{\tau \in \mathbb{R}^{3} \mid \tau_{2}^{2}+\tau_{3}^{2}=\left(L^{2}-\left(\tau_{1}+N\right)^{2}\right)\left(L^{2}-\left(\tau_{1}-N\right)^{2}\right)\right\},
\end{aligned}
$$

for $0 \leq|N| \leq L$ and $L>0$. The invariants $\tau_{2}$ and $\tau_{3}$ lie in the interval $\left[N^{2}-L^{2}, L^{2}-N^{2}\right]$, whereas $\tau_{1}$ belongs to $[|N|-L, L-|N|]$.

It is proved in $[\mathbf{8}, \mathbf{9}]$ that when $0<|N|<L, \mathcal{T}_{L, N}$ is diffeomorphic to the 2-sphere $S^{2}$ and therefore the reduction is regular in that region of the phase space. However, when $N=0$ the space $\mathcal{T}_{L, 0}$ is a topological 2 -sphere with two singular points, namely, the vertices of the surface $\mathcal{T}_{L, 0}$ at $( \pm L, 0,0)$. Thus, the reduction by the axial symmetry is singular $[\mathbf{1}]$. When $|N|=L$ the space $\mathcal{T}_{L, \pm L}$ is just a point. This case corresponds to motions that are circular and equatorial at the same time, and needs to be analyzed on $S^{2} \times S^{2}$, concretely in neighborhoods of the points $(0,0, \pm L, 0,0, \pm L)$. 
The singular points (also called peaks) $( \pm L, 0,0)$ of $\mathcal{T}_{L, 0}$ and the exceptional case of $\mathcal{T}_{L, \pm L}$ are the images of the points $(0,0, \pm L, 0,0, \mp L)$ and $(0,0, \pm L, 0,0, \pm L)$, whereas the rest of the points of $\mathcal{T}_{L, N}$ with $0 \leq|N|<L$ are images of circles on $S^{2} \times S^{2}$ by means of $\pi_{N}$.

It is instructive to stress that as $( \pm L, 0,0)$ are singular points on the orbit space $\mathcal{T}_{L, 0}$, they must be relative equilibria of a reduced system related to a Hamiltonian function, say $\bar{H}$, that is properly defined on these points. Thus, when reconstructing the flow of a certain system whose reduced Hamiltonian is $\bar{H}$ (keeping in mind that $\bar{H}$ has been obtained after reducing the full system by the Keplerian and the axial symmetries), the points $( \pm L, 0,0)$ correspond to families of rectilinear periodic solutions of the full Hamiltonian, that is, they are the same periodic solutions reconstructed from the point $(0,0, \pm L, 0,0, \mp L)$ on $S^{2} \times S^{2}$.

This singular reduction was made for the first time by Cushman in the setting of the artificial satellite theory $[\mathbf{8}]$; see also $[\mathbf{6}]$ and the survey paper $[\mathbf{9}]$.

It is possible to express the quantities $\sin g, \cos g$ and $G$ in terms of $\tau$, $L$, and $N$. Indeed one gets

$$
\begin{aligned}
& 2 G^{2}=L^{2}+N^{2}-\tau_{1}^{2}+\tau_{3} \\
& \cos g=-\frac{\tau_{2}}{\sqrt{\left(L^{2}-N^{2}\right)^{2}-\left(\tau_{1}^{2}-\tau_{3}\right)^{2}}}, \\
& \sin g=\tau_{1} \sqrt{\frac{2\left(L^{2}+N^{2}-\tau_{1}^{2}+\tau_{3}\right)}{\left(L^{2}-N^{2}\right)^{2}-\left(\tau_{1}^{2}-\tau_{3}\right)^{2}}}
\end{aligned}
$$

and this applies when the argument of the perigee $g$ is well defined.

Rectilinear solutions must satisfy $G=N=0$. Taking also into account the constraint appearing in (25), we know that they are defined on the one-dimensional set $\mathcal{R}_{L, 0}=\left\{\tau \in \mathbb{R}^{3} \mid \tau_{2}=0, \tau_{3}=\tau_{1}^{2}-L^{2}\right\}$. Thus, we may analyze linear trajectories as the reduction process regularizes this type of solutions. In particular, the points $( \pm L, 0,0)$ correspond with rectilinear motions such that their projection in the coordinate space leads to rectilinear orbits in the vertical axis $x_{3}$ as the points $( \pm L, 0,0)$ are the projections by $\pi_{N}$ of the points $(0,0, \pm L, 0,0, \mp L)$ on $S^{2} \times S^{2}$. Circular motions are concentrated on a unique point on $\mathcal{T}_{L, N}$ whose coordinates are $\left(0,0, L^{2}-N^{2}\right)$, i.e., on the top point of the orbit space, whereas equatorial (i.e., coplanar with the primaries) trajectories in the twicereduced space are represented on the bottom point of the surface $\mathcal{T}_{L, N}$ with coordinates $\left(0,0, N^{2}-L^{2}\right)$. 
A picture of the twice-reduced space is depicted in Figure 2: the space $\mathcal{T}_{L, N}$ with $N \neq 0$ appears on the top left, the space $\mathcal{T}_{L, 0}$ on the top right, and sections $\tau_{2}=0$ on the lower part. The green points stand for circular solutions, the red points refer to equatorial solutions, and the yellow points denote rectilinear vertical solutions. The lower arc drawn in blue comprise the set of all rectilinear solutions.
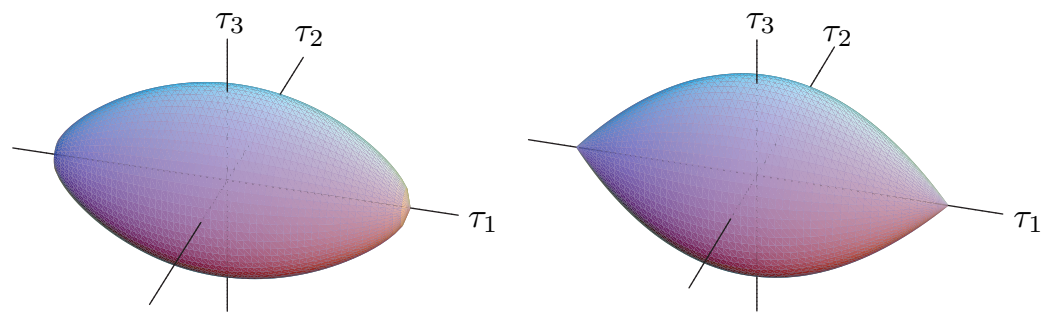

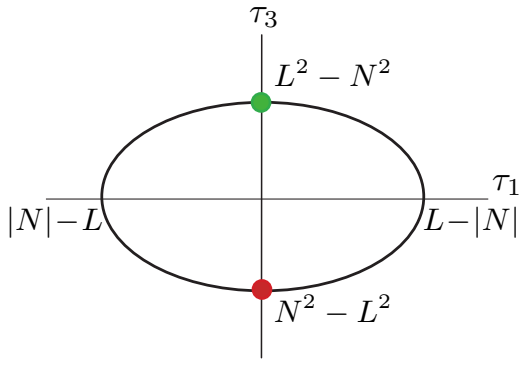

$|N|>0$

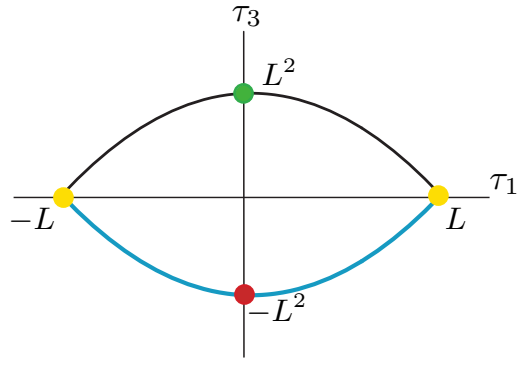

$|N|=0$

Figure 2. Twice-reduced space.

5.2. Dynamics of the spatial lunar problem on $\mathcal{T}_{L, N}$. The normalized Hamiltonian (21) is expressed as a function of the $\tau_{i}$ 's once higher-order terms are omitted. Hence we can remove the zeroth and the $O\left(\varepsilon^{3}\right)$ terms from $\mathcal{H}_{\varepsilon}$. After dropping some constant terms and scaling conveniently, the normal form Hamiltonian $\overline{\mathcal{H}}$ in terms of $\tau$ reads

$$
\overline{\mathcal{H}}=4 \tau_{1}^{2}+\tau_{3}+O\left(\varepsilon^{2}\right) .
$$

The Poisson brackets of the $\tau_{i}$ 's are:

$$
\left\{\tau_{1}, \tau_{2}\right\}=2 \tau_{3}, \quad\left\{\tau_{1}, \tau_{3}\right\}=-2 \tau_{2}, \quad\left\{\tau_{2}, \tau_{3}\right\}=-4 \tau_{1}\left(\tau_{1}^{2}-L^{2}-N^{2}\right)
$$


We can determine the equations of motion related to $\overline{\mathcal{H}}$ by means of the following relations

$$
\dot{\tau}_{i}=\sum_{1 \leq j \leq 3}\left\{\tau_{i}, \tau_{j}\right\} \frac{\partial \overline{\mathcal{H}}}{\partial \tau_{j}}, \quad \text { for } \quad i \in\{1,2,3\} .
$$

Using the Poisson brackets given in (28) we get

$$
\begin{aligned}
& \dot{\tau_{1}}=-2 \tau_{2}, \\
& \dot{\tau_{2}}=-4 \tau_{1}\left(\tau_{1}^{2}+4 \tau_{3}-L^{2}-N^{2}\right), \\
& \dot{\tau_{3}}=16 \tau_{1} \tau_{2} .
\end{aligned}
$$

The relative equilibria are the roots of system (29) that satisfy the constraint of (25) and such that the $\tau_{i}$ 's lie in their appropriate intervals. Their coordinates $\left(\tau_{1}, \tau_{2}, \tau_{3}\right)$ are obtained explicitly, yielding

(i) $\left(0,0, L^{2}-N^{2}\right)$,

(ii) $\left(0,0, N^{2}-L^{2}\right)$,

$$
\text { (iii) }\left( \pm \sqrt{L^{2}+N^{2}-\frac{8 L|N|}{\sqrt{15}}}, 0, \frac{2 L|N|}{\sqrt{15}}\right) \text { with }|N| / L \in[0, \sqrt{3 / 5}] \text {. }
$$

The occurrence of the relative equilibria (i)-(iii) is as follows. We discard the cases $|N|=L$, e.g., the prograde and retrograde circular equatorial solutions, since no dynamics can be analyzed as the orbit space is a point. Moreover, these cases have been already tackled in Subsection 4.2. Thus we will concentrate on the set with $|N|<L$ where $N / L \in(-1,1)$.

We start by noting that the equilibria (i) and (ii) are present for all values of $N$ with $0 \leq|N| \leq L$. The appearance and disappearance of the relative equilibria, when $N / L$ varies, is as follows:

- For $|N| / L \in(\sqrt{3 / 5}, 1)$ the points (i) and (ii) are the only equilibria on the surface $\mathcal{T}_{L, N}$.

- If $|N| / L=\sqrt{3 / 5}$ there are also two equilibria, more concretely the points (ii) and (i) that collides with the points (iii) at $\left(0,0, \frac{2}{5} L^{2}\right)$.

- When $0<|N| / L<\sqrt{3 / 5}$, there are four equilibria, namely the points (i), (ii) and the two points (iii).

- For $N=0$ the coordinates of (iii) become $( \pm L, 0,0)$, that is, they are the singular points of $\mathcal{T}_{L, 0}$. On the other hand the points (i) and (ii) remain as relative equilibria. In particular the point (ii) has coordinates $\left(0,0,-L^{2}\right)$ and it refers to rectilinear solutions. More specifically, they are the solutions whose projections on the coordinate space are straight lines on the $x_{1} x_{2}$-plane passing through the origin with an arbitrary slope. 
Next we summarize the stability character of the relative equilibria although we shall turn to this issue in Subsubsections 5.3.2, 5.3.3 and 5.3.4 when dealing with the KAM 3-tori.

- The point (i) represents motions of circular type with an inclination given by $\cos (I)=N / L$. When $\sqrt{3 / 5}<|N| / L<1$ it is a stable elliptic point (center). If $|N| / L=\sqrt{3 / 5}$ it is a degenerate center with normal form $P^{2}+Q^{4}$, therefore stable. It is a hyperbolic point (saddle) if $0 \leq|N| / L<\sqrt{3 / 5}$.

- The point (ii) corresponds to equatorial trajectories and is elliptic for all $N / L \in(-1,1)$. The eccentricity is given by $\left(1-N^{2} / L^{2}\right)^{1 / 2}$.

- The two relative equilibria corresponding to (iii) represent motions with an inclination given by $\cos (I)= \pm\left(\frac{3}{5}\right)^{1 / 4} \sqrt{|N| / L}$ (the plus sign is for $N \geq 0$ whereas the minus is for $N<0$ ) and an eccentricity $e=\left(1-\left(\frac{5}{3}\right)^{1 / 2}|N| / L\right)^{1 / 2}$. In fact the two equilibrium points (iii) correspond to the same trajectories, but reckoned in a prograde sense for positive $N$ and in a retrograde one for negative $N$. They are elliptic for all $|N| / L \in[0, \sqrt{3 / 5}]$.

In view of the above description a Hamiltonian (supercritical) pitchfork bifurcation of relative equilibria occurs when $N / L$ crosses either the value $-\sqrt{3 / 5}$ or $\sqrt{3 / 5}$ as the two elliptic points (iii) and the hyperbolic one (i) collide. See further details in $[\mathbf{9}, \mathbf{3 2}, \mathbf{3 5}]$.

We present in Figure 3 the flow of the Hamiltonian (27) when $|N| / L$ crosses the value $\sqrt{3 / 5}$. In particular, when $|N| / L \lesssim \sqrt{3 / 5}$, the two equilibria (iii) still exist but are located very close to (i).

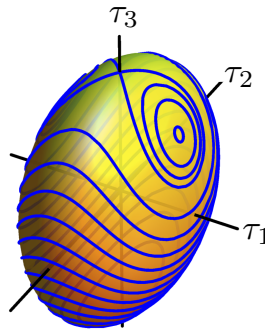

$|N| / L<\sqrt{3 / 5}$

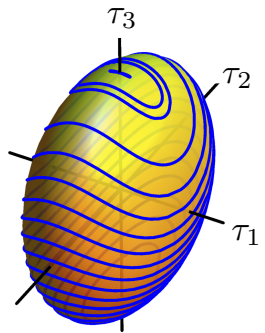

$|N| / L \lesssim \sqrt{3 / 5}$

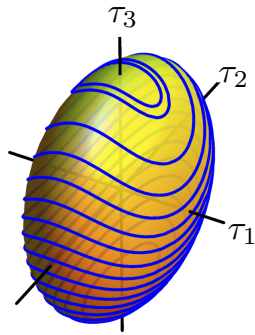

$|N| / L>\sqrt{3 / 5}$

Figure 3. Sequence of the Hamiltonian pitchfork bifurcations that take place when $|N| / L$ passes through $\sqrt{3 / 5}$ 
In Figure 4 we depict the flow of (27) on the twice-reduced space when $N$ is near zero. The space $\mathcal{T}_{L, 0}$ has two singularities at the points $( \pm L, 0,0)$. When $|N|$ tends to zero the points (iii) move on the surface $\mathcal{T}_{L, N}$ away from the point $\left(0,0, L^{2}-N^{2}\right)$ reaching the peaks of $\mathcal{T}_{L, N}$ when $N=0$. The stability of the point (ii) remains the same regardless of the value of $N$ in $(-1,1)$, thus it does not change when $N$ passes through zero.

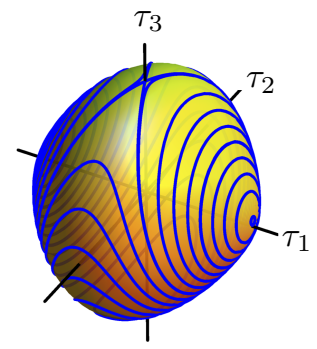

$N \lesssim 0$

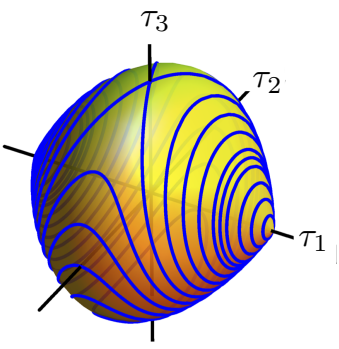

$N=0$

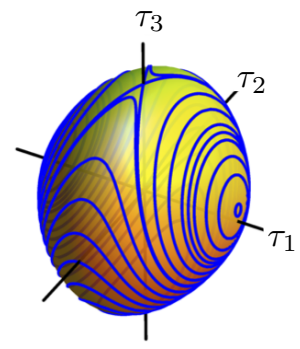

$N \gtrsim 0$

FiguRE 4. The flow of (27) on the twice-reduced space when $N$ is near zero.

We point out that the non-linear stability of the equilibria remains the same as the linear stability excepting for the degenerate cases at the pitchfork bifurcation. The reason is that saddles remain as saddles under perturbation by the Hartman-Grobman Theorem [17] and the linear centers become also non-linear centers since system (29) is of one degree of freedom and the classical Dirichlet stability Theorem applies.

\subsection{Invariant KAM 3-tori from the second reduction.}

\subsubsection{Quasi-periodic solutions of the truncated normal form} Hamiltonian. We have made two processes of normalization, truncation, and reduction (first averaging the mean anomaly and second averaging the argument of the node). So the existence and stability character of the relative equilibria on the orbit space $\mathcal{T}_{L, N}$ are in correspondence with the existence and stability of invariant 2-tori and quasi-periodic solutions of the Hamiltonian (13) in the full phase space once terms of order higher than $O\left(\varepsilon^{6}\right)$ are truncated. Besides, the pitchfork bifurcations occurring at $|N| / L=\sqrt{3 / 5}$ are reconstructed as pitchfork bifurcations of invariant 2-tori of the truncated system (13). However we cannot assure that this dynamics is reflected in the Hamiltonian (12), although we can use the information of some relative equilibria to obtain KAM tori and quasi-periodic solutions for the full system. 
Our goal now is to reconstruct partially the dynamics of the system defined through the Hamiltonian (12) obtaining some new invariant 3-tori and quasi-periodic solutions of the spatial problem using Theorem 2.4. This will be the purpose of Subsubsections 5.3.2, 5.3.3, and 5.3.4. We shall make use of the normal form Hamiltonian (27) and the twicereduced space (25). Besides we shall discuss the existence of the 3-tori whose quasi-periodic solutions are of rectilinear type, a situation already studied in Subsection 4.3. This particular case is associated to the singular points $( \pm L, 0,0)$ and the closed orbits around them. Indeed, these points are reconstructed to the families of periodic solutions studied in Subsection 4.3. We shall see that excepting for the 3-tori related to the peak points we shall need to define local symplectic coordinates in order to be able to construct a pair of action-angle coordinates, say $(J, \varphi)$, so as to apply Han, Li, and Yi's Theorem.

Similar results were obtained by Kummer $[\mathbf{2 1}, \mathbf{2 2}]$ and by Sommer [35]. Kummer performs a second normalization (the one dealing with the axial symmetry) and computes the twice-reduced space but he does not apply singular reduction theory and therefore has to exclude the two singular points that are present when the third component of the angular momentum vanishes. He builds action-angle variables from the twice-reduced space in order to apply KAM Theory and concludes with the existence of some invariant 3 -tori but his results are only partial and his procedure is very intricate.

Sommer also computes the averaged Hamiltonian with respect to the node and uses singular reduction theory. In particular she follows Cushman [9]. Besides she constructs a KAM Theorem to deal with the appearance of the Hamiltonian function at three different time scales and applies her theorem to conclude the persistence of invariant 3-tori around the relative equilibria of (29) that are of elliptic type. Han, Li, and Yi's Theorem generalizes Sommer's Theorem and we use the former together with different sets of symplectic variables, simplifying considerably the amount of computations made by Sommer. Moreover we clarify the existence of the KAM tori that correspond with solutions that are near rectilinear.

In the following we shall focus on the non-degenerate relative equilibria of elliptic type dealt within Subsection 5.2 that are represented by regular or singular points on $\mathcal{T}_{L, N}$. Thus, we concentrate on the point (i) for $|N| / L \in(\sqrt{3 / 5}, 1)$, the point (ii) for $|N| / L \in[0,1)$ and the points (iii) for $|N| / L \in[0, \sqrt{3 / 5})$. 
5.3.2. Invariant 3-tori reconstructed from the point (i). We introduce coordinates

$$
Q=\varepsilon^{-1 / 2} \sqrt{2(L-G)} \cos g, \quad P=\varepsilon^{-1 / 2} \sqrt{2(L-G)} \sin g .
$$

This change is symplectic with multiplier $\varepsilon^{-1}$ and extends analytically to the origin of the $Q P$-plane, provided that $Q$ and $P$ are written in terms of $g$ and $G$ and the computations that carry out satisfy the d'Alembert characteristic; see $[\mathbf{1 9}, \mathbf{2 5}]$ and the examples in [26]. After calculating the inverse of (30) putting $g$ and $G$ in terms of $Q$ and $P$ in the Hamiltonian (21), multiplying this Hamiltonian by $\varepsilon^{-1}$, scaling time to adjust its zeroth term so that it becomes the Kepler Hamiltonian and expanding the result in power series of $\varepsilon$, we end up with

$$
\begin{aligned}
\mathcal{H}_{\varepsilon}= & -\frac{1}{2 L^{2}}-\varepsilon^{3} N+\frac{1}{8} \varepsilon^{6} \mu L^{2}\left(L^{2}-3 N^{2}\right) \\
& -\frac{3}{8} \varepsilon^{7} \mu L\left(2 L^{2} Q^{2}+\left(5 N^{2}-3 L^{2}\right) P^{2}\right)+O\left(\varepsilon^{8}\right) .
\end{aligned}
$$

As $|N| / L \in(\sqrt{3 / 5}, 1)$ it is apparent that the point (i) is elliptic.

The argument of the perigee is undefined for circular motions, however it is straightforward to see that the value of the angle $g$ for points near $\left(0,0, L^{2}-N^{2}\right)$ is close to 0 (for $N>0$ ) or to $\pi$ (for $N<0$ ).

Now we introduce the action-angle pair $(J, \varphi)$ through:

$$
Q=2^{1 / 4} \sqrt{\frac{\sqrt{5 N^{2}-3 L^{2}} J}{L}} \cos \varphi, \quad P=2^{3 / 4} \sqrt{\frac{L J}{\sqrt{5 N^{2}-3 L^{2}}}} \sin \varphi .
$$

The change satisfies $d Q \wedge d P=d J \wedge d \varphi$ and transforms (31) into

$$
\begin{aligned}
\mathcal{H}_{\varepsilon}=-\frac{1}{2 L^{2}}-\varepsilon^{3} N+ & \frac{1}{8} \varepsilon^{6} \mu L^{4}\left(L^{2}-3 N^{2}\right) \\
& -\frac{3 \sqrt{2}}{4} \varepsilon^{7} \mu L^{2} \sqrt{5 N^{2}-3 L^{2}} J+O\left(\varepsilon^{8}\right) .
\end{aligned}
$$

Now we apply Theorem 2.4 with $a=3, m_{1}=3, m_{2}=6, m_{3}=7, n_{0}=1$, $n_{1}=n_{2}=2, n_{3}=3, I^{n_{0}}=(L), I^{n_{1}}=I^{n_{2}}=(L, N), I^{n_{3}}=(L, N, J)$, $\bar{I}^{n_{0}}=(L), \bar{I}^{n_{1}}=\bar{I}^{n_{2}}=(N)$ and $\bar{I}^{n_{3}}=(J)$. One gets

$$
\Omega(I)=\left(\frac{1}{L^{3}},-1,-\frac{3}{4} \mu L^{4} N,-\frac{3 \sqrt{2}}{4} \mu L^{2} \sqrt{5 N^{2}-3 L^{2}}\right)
$$

and the matrix of order 4 whose rows are $\Omega(I), \partial \Omega(I) / \partial L, \partial \Omega(I) / \partial N$, and $\partial \Omega(I) / \partial J$ has rank three. Furthermore, as $b=\sum_{i=1}^{a} m_{i}\left(n_{i}-n_{i-1}\right)$ and the integer $s$ of Theorem 2.4 is 1 , according to [16], the measure 
of excluded tori is of order $O\left(\varepsilon^{b}\right)$, that in this case is $O\left(\varepsilon^{10}\right)$. So we conclude

Proposition 5.1. For $|N| / L \in(\sqrt{3 / 5}, 1)$ there are families of invariant $K A M$ 3-tori filled up by the near circular quasi-periodic solutions of the full system introduced by the Hamiltonian (12). These quasi-periodic solutions have an inclination given approximately by $\cos (I)=N / L$ and a perigee near 0 when $N$ is positive or near $\pi$ when $N$ is negative. The measure of the excluded set of tori is of order $O\left(\varepsilon^{10}\right)$.

5.3.3. Invariant 3-tori reconstructed from the point (ii). We could introduce coordinates based on Delaunay elements, similarly to what is done in Subsubsection 5.3.2, but then the subsequent analysis is not valid for $N=0$. Thus we proceed differently in order to include in the analysis any value of $N$ in $(-1,1)$.

Our aim is to find canonical coordinates $Q, P$ such that the normal form Hamiltonian (21) is written in terms of the new variables (and in terms of $L$ and $N$ ) and we can apply Theorem 2.4. We also require that our approach will be valid for $N=0$ in order to analyze the invariant tori related to motions that are at the same time equatorial and rectilinear. See also the picture (c) of Figure 5 with the circles around the point (ii).

We have represented the flow for the case $N=0$ in Figure 5 from different points of view, showing the appearance of four relative equilibria, namely, three elliptic points and one hyperbolic. The circles surrounding the elliptic points are reconstructed into invariant 3-tori for the spatial lunar problem.

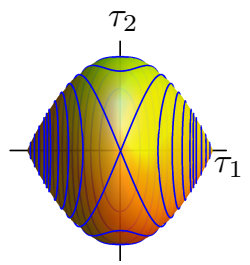

(a)

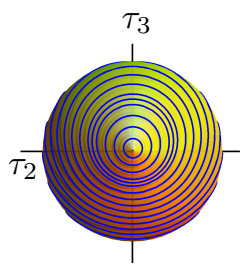

(b)

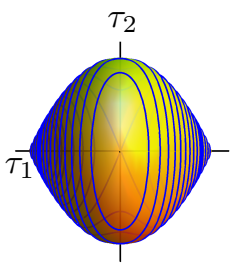

(c)

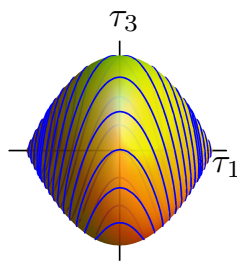

(d)

Figure 5. The flow of (27) for $N=0$ : (a) viewpoint from top, (b) viewpoint from right, (c) viewpoint from bottom and $(\mathrm{d})$ viewpoint from front.

Although the argument of the perigee is not well defined in the case of equatorial motions the value of $g$ for points near $\left(0,0, N^{2}-L^{2}\right)$ is near 0 
(for $N>0$ ) or near $\pi$ (for $N<0$ ). This fact is taken into account when we consider the geometry of the quasi-periodic solutions we will obtain in the next paragraphs.

As we are working in a neighborhood of the point $\left(0,0, N^{2}-L^{2}\right)$ we introduce functions $f_{1}(Q, P ; L, N)$ and $f_{2}(Q, P ; L, N)$, or $f_{1}$ and $f_{2}$ for short, that have to be determined, and are expected to be small. Thus we make the transformation

$$
\tau_{1}=f_{1}, \quad \tau_{2}=f_{2}, \quad \tau_{3}=-\sqrt{f_{1}^{4}-2\left(L^{2}+N^{2}\right) f_{1}^{2}-f_{2}^{2}+\left(L^{2}-N^{2}\right)^{2}},
$$

where we have used the constraint of (25) to put $\tau_{3}$ in terms of $f_{1}$ and $f_{2}$.

Replacing the Poisson brackets between the $\tau_{i}$ 's given in (28) in terms of $Q$ and $P$ and taking into account that $\{Q, P\}=1$ we build three partial differential equations (one for each Poisson bracket) whose unknowns are $f_{1}$ and $f_{2}$. Out of the three equations there is an essential expression that has to be zero, the other equations being redundant. The relevant equation is

$$
2 \sqrt{f_{1}^{4}-2\left(L^{2}+N^{2}\right) f_{1}^{2}-f_{2}^{2}+\left(L^{2}-N^{2}\right)^{2}}+\frac{\partial f_{1}}{\partial Q} \frac{\partial f_{2}}{\partial P}-\frac{\partial f_{1}}{\partial P} \frac{\partial f_{2}}{\partial Q}=0 .
$$

As we can choose either $f_{1}$ or $f_{2}$ we select a convenient value for $f_{1}=P$ and solve for $f_{2}$, getting

$$
\begin{aligned}
& f_{1}(Q, P ; L, N)=P \\
& f_{2}(Q, P ; L, N)=\sqrt{\left((L+N)^{2}-P^{2}\right)\left((L-N)^{2}-P^{2}\right)} \sin (2 Q) .
\end{aligned}
$$

We note that $Q=P=0$ implies $f_{1}=f_{2}=0$ and $\tau_{1}=\tau_{2}=0$ while $\tau_{3}=N^{2}-L^{2}$; besides when $Q$ and $P$ are small, $f_{1}$ and $f_{2}$ are small quantities.

Once $f_{1}$ and $f_{2}$ are calculated the final change is

$$
\begin{aligned}
& \tau_{1}=P, \\
& \tau_{2}=\sqrt{\left((L+N)^{2}-P^{2}\right)\left((L-N)^{2}-P^{2}\right)} \sin (2 Q), \\
& \tau_{3}=-\sqrt{\left((L+N)^{2}-P^{2}\right)\left((L-N)^{2}-P^{2}\right)} \cos (2 Q) .
\end{aligned}
$$

The equation $\tau_{1}=P$ represents the local surface around the equilibrium $Q=P=0$ in the space $Q P \tau_{1}$, which means that $\left(0,0, N^{2}-L^{2}\right)$ is a regular point in this chart. In fact we are projecting the Hamiltonian function in the $Q P$-plane as the change (33) is valid only in a neighborhood of the point $\left(0,0, N^{2}-L^{2}\right)$ on $\mathcal{T}_{L, N}$.

In particular when $N=0$ the above transformation reduces to

$$
\tau_{1}=P, \quad \tau_{2}=\left(L^{2}-P^{2}\right) \sin (2 Q), \quad \tau_{3}=-\left(L^{2}-P^{2}\right) \cos (2 Q) .
$$


Next we put $g, \sin g$ and $\cos g$ in terms of $Q$ and $P$ by means of (26), yielding the expression

$$
\begin{aligned}
& 2 G^{2}=L^{2}+N^{2}-P^{2}-\sqrt{\left((L+N)^{2}-P^{2}\right)\left((L-N)^{2}-P^{2}\right)} \cos (2 Q), \\
& \cos g=-\frac{\sqrt{\left((L+N)^{2}-P^{2}\right)\left((L-N)^{2}-P^{2}\right)} \sin (2 Q)}{\sqrt{\left(L^{2}-N^{2}\right)^{2}-\left(P^{2}+\sqrt{\left((L+N)^{2}-P^{2}\right)\left((L-N)^{2}-P^{2}\right)} \cos (2 Q)\right)^{2}}}, \\
& \sin g=\sqrt{2} P \frac{\sqrt{L^{2}+N^{2}-P^{2}-\sqrt{\left((L+N)^{2}-P^{2}\right)\left((L-N)^{2}-P^{2}\right)} \cos (2 Q)}}{\sqrt{\left(L^{2}-N^{2}\right)^{2}-\left(P^{2}+\sqrt{\left((L+N)^{2}-P^{2}\right)\left((L-N)^{2}-P^{2}\right)} \cos (2 Q)\right)^{2}}},
\end{aligned}
$$

which is a valid expression except for the case of equatorial motions, i.e., when $Q=P=0$ as then $G=|N|$ and $g$ is undefined. The Hamiltonian (21) is put in terms of $Q$ and $P$ using (36). Next we scale $Q$ and $P$ by doing $(\bar{Q}, \bar{P})=\varepsilon^{1 / 2}(Q, P)$, multiply $\mathcal{H}_{\varepsilon}$ by the multiplier $\varepsilon^{-1}$ and scale time conveniently. After expanding the Hamiltonian in powers of $\varepsilon$ and dropping constant terms we obtain:

$$
\begin{aligned}
\mathcal{H}_{\varepsilon}= & -\frac{1}{2 L^{2}}-\varepsilon^{3} N-\frac{1}{8} \varepsilon^{6} \mu L^{2}\left(5 L^{2}-3 N^{2}\right) \\
& +\frac{3}{8} \varepsilon^{7} \mu L^{2}\left(2\left(L^{2}-N^{2}\right) \bar{Q}^{2}+\frac{5 L^{2}-3 N^{2}}{L^{2}-N^{2}} \bar{P}^{2}\right)+O\left(\varepsilon^{8}\right) .
\end{aligned}
$$

The development is valid for $0 \leq|N|<L$, and looking at the term factorized by $\varepsilon^{7}$, the point (ii) is elliptic even for $N=0$ as was already stated in Subsection 5.2.

The next step is the introduction of an action-angle pair $(J, \varphi)$ :

$$
\bar{Q}=2^{1 / 4} \sqrt{\frac{\sqrt{5 L^{2}-3 N^{2}} J}{L^{2}-N^{2}}} \cos \varphi, \quad \bar{P}=2^{3 / 4} \sqrt{\frac{\left(L^{2}-N^{2}\right) J}{\sqrt{5 L^{2}-3 N^{2}}}} \sin \varphi,
$$

transforming (37) into

$$
\begin{aligned}
\mathcal{H}_{\varepsilon}=-\frac{1}{2 L^{2}}-\varepsilon^{3} N- & \frac{1}{8} \varepsilon^{6} \mu L^{2}\left(5 L^{2}-3 N^{2}\right) \\
& +\frac{3 \sqrt{2}}{4} \varepsilon^{7} \mu L^{2} \sqrt{5 L^{2}-3 N^{2}} J+O\left(\varepsilon^{8}\right) .
\end{aligned}
$$


Note that $d \bar{Q} \wedge d \bar{P}=d J \wedge d \varphi$. Next one can apply Theorem 2.4 where the values of $a, m_{j}$ 's, $n_{j}$ 's, $I^{n_{j}}$ 's, $\bar{I}^{n_{j}}$ 's are as in Subsubsection 5.3.2. We obtain

$$
\Omega(I)=\left(\frac{1}{L^{3}},-1, \frac{3}{4} \mu L^{2} N, \frac{3 \sqrt{2}}{4} \mu L^{2} \sqrt{5 L^{2}-3 N^{2}}\right),
$$

and the corresponding matrix of order 4 whose rows are given by $\Omega(I)$, $\partial \Omega(I) / \partial L, \partial \Omega(I) / \partial N$, and $\partial \Omega(I) / \partial J$ has rank three. We get

Proposition 5.2. There are families of invariant KAM 3-tori filled up by the near equatorial quasi-periodic solutions of the full system introduced by the Hamiltonian (12). These quasi-periodic solutions have an eccentricity given approximately by $\left(1-N^{2} / L^{2}\right)^{1 / 2}$ where $|N| / L \in[0,1)$ and a perigee close to 0 (for positive $N$ ) or $\pi$ (for negative $N$ ). When $N \approx 0$ these quasi-periodic motions are near rectilinear equatorial solutions. The measure of the excluded tori is of order $O\left(\varepsilon^{10}\right)$.

The computations carried out are valid for all $N$, thus we can conclude that the KAM 3-tori also exist for $N$ near zero. This fact is not easily deductible from the exposition made by Sommer [35] in Chapter 5 of her thesis.

5.3.4. Invariant 3-tori reconstructed from the points (iii). We consider the points (iii) with $|N| / L \in[0, \sqrt{3 / 5}$ ). The corresponding Delaunay coordinates for these equilibria are $\left(g_{0}, G_{0}\right)=\left( \pm \pi / 2,(5 / 3)^{1 / 4} \sqrt{L|N|}\right)$. In particular, when $N=0$ it implies that the Delaunay coordinates for the points $( \pm L, 0,0)$ on the surface $\mathcal{T}_{L, 0}$ are $\left(g_{0}, G_{0}\right)=( \pm \pi / 2,0)$. Note that even when the argument of the perigee is not properly defined for rectilinear motions and so we cannot say that $g_{0}$ takes any value when $G=0$, it is of interest to note that the corresponding quasi-periodic solutions that will be reconstructed from these points will have perigees near $\pm \pi / 2$ (specifically $\pi / 2$ when $N>0$ and $-\pi / 2$ if $N<0$ ).

We start by considering the case $N \neq 0$ as the subsequent analysis is not valid when $N$ vanishes.

When $|N|>0$ Delaunay elements may be used as they are a well defined set of coordinates. In fact an adequate transformation is defined by

$$
Q=\varepsilon^{-1 / 2}\left(g-g_{0}\right), \quad P=\varepsilon^{-1 / 2}\left(G-G_{0}\right) .
$$

This change is canonical with multiplier $\varepsilon^{-1}$. We put $g$ and $G$ in terms of $Q, P, g_{0}$, and $G_{0}$, then we multiply $\mathcal{H}_{\varepsilon}$ by $\varepsilon^{-1}$ and scale time. Finally 
we expand the Hamiltonian in power series of $\varepsilon$ around zero, getting

$$
\begin{aligned}
\mathcal{H}_{\varepsilon}= & -\frac{1}{2 L^{2}}-\varepsilon^{3} N+\frac{5}{4} \varepsilon^{6} \mu L^{2}(L-\sqrt{3 / 5}|N|)(L-2 \sqrt{3 / 5}|N|) \\
& -\frac{1}{8} \varepsilon^{7} \mu L^{2}\left(15(L-\sqrt{3 / 5}|N|)(L-\sqrt{5 / 3}|N|) Q^{2}+36 P^{2}\right) \\
& +O\left(\varepsilon^{15 / 2}\right) .
\end{aligned}
$$

Moreover higher-order terms are unbounded for $N=0$, what prevents the discussion of these paragraphs to be extended to $N=0$ or small. Thus for the moment we assume that $|N| \geq N^{*}$ for some $N^{*}>0$ such that the ordering established through the small parameter $\varepsilon$ remains the same. We shall deal with the case $N \approx 0$ later on.

As $|N| / L \in\left[N^{*} / L, \sqrt{3 / 5}\right.$ ) the points (iii) are elliptic in agreement with Subsection 5.2. Indeed, the points (iii) are also elliptic when $|N| / L=$ $\sqrt{3 / 5}$ but then $G_{0}=L$ and the coordinates (39) are not adequate to handle the stability analysis and to obtain the related invariant 3 -tori. However it is not a problem because, when $|N| / L=\sqrt{3 / 5}$, the points (iii) and the point (ii) coincide and we have analyzed its stability character, obtaining as well the KAM 3-tori in Proposition 5.2.

We introduce the action-angle variables $(J, \varphi)$ by means of

$$
\begin{aligned}
& Q=2 \sqrt{3} \sqrt{\frac{J}{\sqrt{15(L-\sqrt{3 / 5}|N|)(L-\sqrt{5 / 3}|N|)}}} \cos \varphi, \\
& P=\frac{1}{\sqrt{3}} \sqrt{\sqrt{15(L-\sqrt{3 / 5}|N|)(L-\sqrt{5 / 3}|N|)}} J \sin \varphi,
\end{aligned}
$$

satisfying $d Q \wedge d P=d J \wedge d \varphi$. This allows us to transform (40) into

$$
\begin{aligned}
\mathcal{H}_{\varepsilon}= & -\frac{1}{2 L^{2}}-\varepsilon^{3} N+\frac{5}{4} \varepsilon^{6} \mu L^{2}(L-\sqrt{3 / 5}|N|)(L-2 \sqrt{3 / 5}|N|) \\
& -\frac{3}{2} \varepsilon^{7} \mu L^{2} \sqrt{15(L-\sqrt{3 / 5}|N|)(L-\sqrt{5 / 3}|N|)} J+O\left(\varepsilon^{15 / 2}\right) .
\end{aligned}
$$

In order to apply Theorem 2.4 we note that the values of the constants needed in the hypotheses of the theorem are as in the previous cases. 
This time the frequency vector is

$$
\begin{aligned}
\Omega(I)=\left(\frac{1}{L^{3}},-1, \frac{3}{4} \mu\right. & L^{2}(\mp \sqrt{15} L+4 N), \\
& \left.-\frac{3}{2} \mu L^{2} \sqrt{15(L-\sqrt{3 / 5}|N|)(L-\sqrt{5 / 3}|N|)}\right),
\end{aligned}
$$

the minus sign in the formula applies for $N>0$ while the plus sign applies for $N<0$. The matrix of order 4 whose rows are $\Omega(I), \partial \Omega(I) / \partial L$, $\partial \Omega(I) / \partial N$, and $\partial \Omega(I) / \partial J$ has rank three.

Now we focus on the case $N \approx 0$ (i.e. the case $0 \leq|N|<N^{*}$ ), that is, we analyze the stability of the points (iii) for $N$ small and on the singular points $( \pm L, 0,0)$ for $N=0$ and the existence of the related KAM 3-tori. We scale the normalized Hamiltonian (21) using $N=\varepsilon^{4} N$. Then $\mathcal{H}_{\varepsilon}$ is transformed into

$$
\mathcal{H}_{\varepsilon}=-\frac{1}{2 L^{2}}+\frac{1}{16} \varepsilon^{6} \mu L^{2}\left(5 L^{2}-3 G^{2}-15\left(L^{2}-G^{2}\right) \cos (2 g)\right)+O\left(\varepsilon^{7}\right)
$$

where the higher order tems contain the scaled action $N$.

Omitting higher-order terms we find that the Hamiltonian (42) is the same as (27) when we take $N=0$, i.e., the Hamiltonian defined on $\mathcal{T}_{L, 0}$. Note that the procedure applies for non-null but small $N$ as the above scaling makes that the case $N \approx 0$ is the same as the case $N=0$ up to terms of order seven in $\varepsilon$.

See the four pictures of Figure 5 and the picture in the middle of Figure 4 with the closed orbits encircling the points $( \pm L, 0,0)$.

Inspired in the way it was done in [15, Subsection 4.4] we introduce a transformation to desingularize the points $( \pm L, 0,0)$ as follows:

$$
\tau_{1}=\frac{1}{2} w, \quad \tau_{2}=\frac{1}{4}\left(u^{2}-v^{2}\right), \quad \tau_{3}=\frac{1}{2} u v .
$$

This change is a $2: 1$-covering and transforms the constraint (25) defining the space $\mathcal{T}_{L, 0}$ into the 2 -sphere

$$
\mathcal{Q}_{L, 0}=u^{2}+v^{2}+w^{2}-4 L^{2}=0 .
$$

Thus the points $( \pm L, 0,0)$ are converted into the points $(u, v, w)=$ $(0,0, \pm 2 L)$ and these points become regular points of $\mathcal{Q}_{L, 0}$. The transformation (43) is a change of variables that applies in the neighborhood of the points (iii) for $|N|$ smaller than $N^{*}$ or null.

After removing constant terms and projecting the Hamiltonian into the $u v$-plane, the normal form Hamiltonian (27) is

$$
\overline{\mathcal{H}}=\frac{1}{2} u v-\left(u^{2}+v^{2}\right)+\cdots .
$$


The pair $(u, v)$ does not form a symplectic chart, indeed the Poisson bracket between the two is computed as the vector triple product $\{u, v\}=$ $(\nabla u \times \nabla v) \cdot \nabla \mathcal{Q}_{L, 0}$ where $\nabla$ denotes the gradient of a scalar function in the chart $(u, v, w)$. We get

$$
\{u, v\}= \pm \frac{1}{2} \sqrt{4 L^{2}-u^{2}-v^{2}},
$$

where the plus sign applies for $(L, 0,0)$ while the minus is used for $(-L, 0,0)$.

The equations of motion associated to (44) are

$$
\begin{aligned}
& \dot{u}=\{u, v\} \frac{\partial \overline{\mathcal{H}}}{\partial v}= \pm \frac{1}{4}(u-4 v) \sqrt{4 L^{2}-u^{2}-v^{2}}, \\
& \dot{v}=-\{u, v\} \frac{\partial \overline{\mathcal{H}}}{\partial u}= \pm \frac{1}{4}(4 u-v) \sqrt{4 L^{2}-u^{2}-v^{2}} .
\end{aligned}
$$

The point $\left(u_{0}, v_{0}\right)=(0,0)$ is a critical point of (45) that corresponds to $( \pm L, 0,0)$ on $\mathcal{T}_{L, 0}$.

The eigenvalues associated with the linearized equations around $\left(u_{0}, v_{0}\right)$ are $\pm \sqrt{15} L i$. Thus the points $(0,0, \pm 2 L)$ are stable, hence $( \pm L, 0,0)$ are stable points on $\mathcal{T}_{L, 0}$, which is equivalent to the fact that the points $(0,0, \pm L, 0,0, \mp L)$ are stable points on $S^{2} \times S^{2}$. The existence of the relative equilibria $( \pm L, 0,0)$ leads to the existence of two families of elliptic periodic solutions for the full system (12) that are of rectilinear type, these solutions already analyzed in Subsection 4.3. We can say more, e.g., that the points (iii) are also stable for $N$ small, which is in agreement with the stability character of (iii) for $N$ such that $N^{*} / L \leq|N| / L<\sqrt{3 / 5}$.

Finally in order to establish the existence of KAM 3-tori when $N \approx 0$ for the full system we can use the result of Proposition 4.3 after undoing the scaling $N=\varepsilon^{4} N$ and the transformation to normal form that led to (21), we go back to the Hamiltonian in normal form given by (15). Thus repeating the steps of Subsection 4.3 and using Proposition 4.3, we end up with the existence of KAM 3-tori surrounding the periodic solutions that are near rectilinear and whose projections in the coordinate space indicate that they move close to the axis $x_{3}$ up and down. This implies that there are KAM 3-tori around the points (iii) for small $N$ (i.e., $\left.|N|<N^{*}\right)$ and around the points $( \pm L, 0,0)$ for $N=0$. In conclusion there are invariant 3 -tori related to the points (iii) for all $N$ with $0<|N| / L<\sqrt{3 / 5}$ and there are invariant 3 -tori related to the points $( \pm L, 0,0)$ for $N=0$. In summary: 
Proposition 5.3. There are families of invariant KAM 3-tori filled with quasi-periodic solutions of the full system introduced by the Hamiltonian (12). These quasi-periodic solutions have approximate inclination and eccentricity given respectively by $\cos (I)= \pm\left(\frac{3}{5}\right)^{1 / 4} \sqrt{|N| / L}$ (the plus sign applies for $N \geq 0$ while the minus sign applies for $N<0$ ) and $\left(1-\left(\frac{5}{3}\right)^{1 / 2}|N| / L\right)^{1 / 2}$ where $|N| / L \in[0, \sqrt{3 / 5})$. The perigee of these quasi-periodic solutions is near $\pi / 2$ for positive $N$ and near $-\pi / 2$ for negative $N$. The measure of the excluded tori is of order $O\left(\varepsilon^{10}\right)$.

In the estimate of the measure of the excluded tori in Proposition 5.3 we have chosen the exponent ten as it is the minimum of the exponents of Proposition 4.3 and the one corresponding to the Hamiltonian (41), which is twelve.

\section{Acknowledgments}

The authors are partially supported by the Spanish MINECO grant MTM 2011-28227-C02-01 and by the Charles Phelps Taft Foundation.

\section{References}

[1] J. M. Arms, R. H. Cushman, and M. J. Gotay, A universal reduction procedure for Hamiltonian group actions, in: "The geometry of Hamiltonian systems" (Berkeley, CA, 1989), Math. Sci. Res. Inst. Publ. 22, Springer, New York, 1991, pp. 33-51. DOI: 10.1007/978-1-4613-9725-0_4.

[2] V. I. Arnold, V. V. Kozlov, And A. I. NeǏshtadt, "Mathematical aspects of classical and celestial mechanics", [Dynamical systems. III], Translated from the Russian original by E. Khukhro, Third edition, Encyclopaedia of Mathematical Sciences 3, SpringerVerlag, Berlin, 2006.

[3] E. A. Belbruno, A new family of periodic orbits for the restricted problem, Celestial Mech. 25(2) (1981), 195-217. DOI: 10.1007/BF01230520.

[4] A. Chenciner And J. Llibre, A note on the existence of invariant punctured tori in the planar circular restricted three-body problem, Ergodic Theory Dynam. Systems 8* (1988), Charles Conley Memorial Issue, 63-72. DOI: 10.1017/S0143385700009330.

[5] R. C. Churchill and M. Kummer, A unified approach to linear and nonlinear normal forms for Hamiltonian systems, J. Symbolic Comput. 27(1) (1999), 49-131. DOI: 10.1006/jsco.1998.0244. 
[6] S. L. Coffey, A. Deprit, And B. R. Miller, The critical inclination in artificial satellite theory, Celestial Mech. 39(4) (1986), 365-406. DOI : $10.1007 / B F 01230483$.

[7] C. Conley, On some new long periodic solutions of the plane restricted three body problem, Comm. Pure Appl. Math. 16 (1963), 449-467. DOI: 10.1002/cpa.3160160405.

[8] R. Cushman, Reduction, Brouwer's Hamiltonian, and the critical inclination, Celestial Mech. 31(4) (1983), 401-429. DOI: 10.1007/BF01230294.

[9] R. H. Cushman, A survey of normalization techniques applied to perturbed Keplerian systems, in: "Dynamics reported: expositions in dynamical systems", Dynam. Report. Expositions Dynam. Systems (N.S.) 1, Springer, Berlin, 1992, pp. 54-112.

[10] R. H. Cushman And J. J. DuistermaAt, A characterization of the Ligon-Schaaf regularization map, Comm. Pure Appl. Math. 50(8) (1997), 773-787. DOI: 10.1002/(SICI) 1097-0312(199708)50: 8<773: :AID-CPA3>3.0. CO ; 2-3.

[11] A. Deprit, Canonical transformations depending on a small parameter, Celestial Mech. 1 (1969/1970), 12-30. DOI: 10.1007/ BF01230629.

[12] A. Deprit, The elimination of the parallax in satellite theory, $\mathrm{Ce}$ lestial Mech. 24(2) (1981), 111-153. DOI : 10.1007/BF01229192.

[13] A. Deprit, Delaunay normalisations, Celestial Mech. 26(1) (1982), 9-21. DOI: $10.1007 / \mathrm{BF} 01233178$.

[14] G. L. Dirichlet, Über die Stabilität des Gleichgewichts, J. Reine Angew. Math. 32 (1846), 85-88. DOI: 10.1515/crll.1846.32.85.

[15] S. Ferrer, H. Hanssmann, J. Palacián, and P. Yanguas, On perturbed oscillators in 1-1-1 resonance: the case of axially symmetric cubic potentials, J. Geom. Phys. 40(3-4) (2002), 320-369. DOI: 10.1016/S0393-0440(01) 00041-9.

[16] Y. HAN, Y. LI, AND Y. YI, Invariant tori in Hamiltonian systems with high order proper degeneracy, Ann. Henri Poincaré 10(8) (2010), 1419-1436. DOI: 10.1007/s00023-010-0026-7.

[17] P. Hartman, "Ordinary differential equations", John Wiley \& Sons, Inc., New York-London-Sydney, 1964.

[18] G. Heckman and T. DE LAat, On the regularization of the Kepler problem, J. Symplectic Geom. 10(3) (2012), 463-473.

[19] J. HENRARD, Virtual singularities in the artificial satellite theory, Celestial Mech. 10 (1974), 437-449. DOI : 10.1007/BF01229120.

[20] M. Iñarrea, V. Lanchares, J. F. Palacián, A. Pascual, J. P. SAlas, And P. Yanguas, Symplectic coordinates on $S^{2} \times S^{2}$ 
for perturbed Keplerian problems: application to the dynamics of a generalised Størmer problem, J. Differential Equations 250(3) (2011), 1386-1407. DOI: 10.1016/j.jde.2010.09.027.

[21] M. Kummer, On the three-dimensional lunar problem and other perturbation problems of the Kepler problem, J. Math. Anal. Appl. 93(1) (1983), 142-194. DOI : 10.1016/0022-247X (83) 90224-X.

[22] M. Kummer, Reduction in the rotating Kepler problem and related topics, in: "Hamiltonian dynamics and celestial mechanics" (Seattle, WA, 1995), Contemp. Math. 198, Amer. Math. Soc., Providence, RI, 1996, pp. 155-179. DOI: 10.1090/conm/198/02488.

[23] A. J. Laub and K. Meyer, Canonical forms for symplectic and Hamiltonian matrices, Celestial Mech. 9 (1974), 213-238. DOI: 10.1007/BF01260514.

[24] T. Ligon And M. SchaAf, On the global symmetry of the classical Kepler problem, Rep. Mathematical Phys. 9(3) (1976), 281-300.

[25] K. R. Meyer, G. R. Hall, And D. Offin, "Introduction to Hamiltonian dynamical systems and the $N$-body problem", Second edition, Applied Mathematical Sciences 90, Springer, New York, 2009.

[26] K. R. Meyer, J. F. Palacián, and P. Yanguas, Geometric averaging of Hamiltonian systems: periodic solutions, stability, and KAM tori, SIAM J. Appl. Dyn. Syst. 10(3) (2011), 817-856. DOI: 10.1137/100807673.

[27] K. R. Meyer And D. S. Schmidt, The stability of the Lagrange triangular point and a theorem of Arnol'd, J. Differential Equations 62(2) (1986), 222-236. DOI : 10.1016/0022-0396 (86) 90098-7.

[28] J. Moser, On invariant curves of area-preserving mappings of an annulus, Nachr. Akad. Wiss. Göttingen Math.-Phys. Kl. II 1962 (1962), 1-20.

[29] J. Moser, Regularization of Kepler's problem and the averaging method on a manifold, Comm. Pure Appl. Math. 23(4) (1970), 609-636. DOI: $10.1002 / \mathrm{cpa} .3160230406$.

[30] J. Moser, "Stable and random motions in dynamical systems", With special emphasis on celestial mechanics, Hermann Weyl Lectures, the Institute for Advanced Study, Princeton, N. J. Annals of Mathematics Studies 77, Princeton University Press, Princeton, N. J.; University of Tokyo Press, Tokyo, 1973.

[31] J. PAlacián, Normal forms for perturbed Keplerian systems, $J$. Differential Equations 180(2) (2002), 471-519. DOI: 10.1006/jdeq. 2001.4068. 
[32] J. Palacián and P. Yanguas, Invariant manifolds of spatial restricted three-body problems: the lunar case, in: "New advances in celestial mechanics and Hamiltonian systems", Kluwer/Plenum, New York, 2004, pp. 199-224.

[33] G. Reeb, Sur certaines propriétés topologiques des trajectoires des systèmes dynamiques, Acad. Roy. Belgique. Cl. Sci. Mém. Coll. in $8^{\circ} \mathbf{2 7}$ (9) (1952), 64 pp.

[34] C. L. Siegel And J. K. Moser, "Lectures on celestial mechanics", Translation by Charles I. Kalme, Die Grundlehren der mathematischen Wissenschaften 187, Springer-Verlag, New York-Heidelberg, 1971.

[35] B. Sommer, A KAM Theorem for the spatial lunar problem, Ph.D. Thesis, University of Aachen, Germany (2003).

[36] P. Yanguas, J. F. Palacián, K. R. Meyer, and H. S. Dumas, Periodic solutions in Hamiltonian systems, averaging, and the lunar problem, SIAM J. Appl. Dyn. Syst. 7(2) (2008), 311-340. DOI: 10.1137/070696453.

Kenneth R. Meyer:

Department of Mathematical Sciences

University of Cincinnati

Cincinnati, Ohio 45221-0025

USA

E-mail address: ken.meyer@uc.edu

Jesús F. Palacián and Patricia Yanguas:

Departamento de Ingeniería Matemática e Informática

Universidad Pública de Navarra

31006 Pamplona

Spain

E-mail address: palacian@unavarra.es

E-mail address: yanguas@unavarra.es 